\title{
Germination Stimulants of Phelipanche ramosa in the Rhizosphere of Brassica napus Are Derived from the Glucosinolate Pathway
}

\author{
Bathilde Auger, ${ }^{1}$ Jean-Bernard Pouvreau, ${ }^{1}$ Karinne Pouponneau, ${ }^{2}$ Kaori Yoneyama, ${ }^{3}$ Grégory Montiel, ${ }^{1}$ \\ Bruno Le Bizec, ${ }^{2}$ Koichi Yoneyama, ${ }^{3}$ Philippe Delavault, ${ }^{1}$ Régine Delourme, ${ }^{4}$ and Philippe Simier ${ }^{1}$ \\ ${ }^{1}$ LUNAM Université, Laboratoire de Biologie et Pathologie Végétales, SFR 4207 QUASAV, UFR Sciences et Techniques, \\ F-44322 Nantes, France; ${ }^{2}$ LUNAM Université, Oniris, LABERCA, F-44307 Nantes, France; ${ }^{3}$ Utsunomiya University, Weed \\ Science Center, Utsunomiya 321-8505, Japan; ${ }^{4}$ INRA, UMR1349 IGEPP, F-35653 Le Rheu, France
}

Submitted 16 January 2012. Accepted 8 March 2012.

\begin{abstract}
Phelipanche ramosa is a major parasitic weed of Brassica napus. The first step in a host-parasitic plant interaction is stimulation of parasite seed germination by compounds released from host roots. However, germination stimulants produced by $B$. napus have not been identified yet. In this study, we characterized the germination stimulants that accumulate in $B$. napus roots and are released into the rhizosphere. Eight glucosinolate-breakdown products were identified and quantified in $B$. napus roots by gas chromatography-mass spectrometry. Two (3-phenylpropanenitrile and 2-phenylethyl isothiocyanate [2-PEITC]) were identified in the $B$. napus rhizosphere. Among glucosinolatebreakdown products, $P$. ramosa germination was strongly and specifically triggered by isothiocyanates, indicating that 2-PEITC, in particular, plays a key role in the B. napus$P$. ramosa interaction. Known strigolactones were not detected by ultraperformance liquid chromatography-tandem mass spectrometry, and seed of Phelipanche and Orobanche spp. that respond to strigolactones but not to isothiocyanates did not germinate in the rhizosphere of $B$. napus. Furthermore, both wild-type and strigolactone biosynthesis mutants of Arabidopsis thaliana Atccd7 and Atccd8 induced similar levels of $P$. ramosa seed germination, suggesting that compounds other than strigolactone function as germination stimulants for $P$. ramosa in other Brassicaceae spp. Our results open perspectives on the high adaptation potential of root-parasitic plants under host-driven selection pressures.
\end{abstract}

The root-parasitic plants Orobanche and Phelipanche spp. can cause devastating yield reductions in many important crops throughout the world, such as legumes, crucifers, sunflower, tobacco, and tomato (Parker 2009). Of the non-chlorophyllous parasitic plants, major pests include the weedy Orobanchaceae Phelipanche ramosa L. Pomel, P. aegyptiaca (Pers.) Pomel, and Orobanche crenata Forsk., all of which have para-

Corresponding author: P. Simier; Telephone: +33-2-51125616; Fax: +332-51125612; E-mail: philippe.simier@univ-nantes.fr

* The $\boldsymbol{e}$-Xtra logo stands for "electronic extra" and indicates that a supplementary table is published online.

This article is in the public domain and not copyrightable. It may be freely reprinted with customary crediting of the source. The American Phytopathological Society, 2012. sitized species of agronomic interest since antiquity, as well as $O$. cumana Wallr. and $O$. foetida Poir., which have emerged as weeds more recently (Musselman 1980; Parker 2009). These species are holoparasites and depend entirely on their host for nutrients and water, which are taken up from the host phloem through a haustorium. These Orobanchaceae spp. have an annual lifecycle and can be found mainly in artificial or human-disturbed environments, where host predictability is relatively low. Seed germination in these species is stimulated by the perception of secondary metabolites released from the roots of a potential host plant. Therefore, to identify host roots in the immediate vicinity, weedy species must be sensitive enough to germination stimulants to detect them at very low concentrations. Furthermore, most of these weedy parasites have a wide host range and geographic distribution (Parker 2009). To overcome the problem of suicidal germination in the absence of a proper host plant, these parasites also produce large quantities of seed, which can remain viable for decades.

Among these parasites, P. ramosa is by far the most widespread, because its habitat covers central and southern Europe, northern Africa, Asia, and, more recently, Central America and Australia (Parker 2009). In central Europe, P. ramosa has become established in long-season winter crops, as well as in short-season spring and summer crops such as hemp, melon, tobacco, tomato, and potato (Parker and Riches 1993). It has also recently become a major parasitic weed of winter-type oilseed rape (Brassica napus L.) and a virulent population has spread all over western France (Brault et al. 2007; Gibot-Leclerc et al. 2003). To overcome this growing problem, several strategies have been proposed, such as control methods based on solarization, soil management, the use of chemicals, trap crops, and breeding for resistant hosts. However, due to the huge number of long-viable parasite seed, individual control measures are effective only to a limited extent and these strategies must be implemented in a coordinated manner to efficiently maintain crop yields at levels similar to those reached in noninfected areas. Host resistance is generally considered to be a key component of integrated pest management and can operate at different stages of the parasite lifecycle (Perez de Luque et al. 2009; Rispail et al. 2007; Yoder and Scholes 2010). The first event in the interaction between parasitic plants and their hosts is the stimulation of parasite seed germination, which is triggered only by compounds released from host roots. It has been shown that host plants producing lower amounts of germination stimulants are less infected by Orobanchaceae plants (Gomez-Roldan et al. 2008; Umehara et al. 2008). Thus, many breeding strategies 
have focused on the reduction of production and exudation of germination stimulants (Lopez-Raez et al. 2009; Rispail et al. 2007; Rubiales and Fernandez-Aparicio 2011). However, in the case of the $B$. napus $-P$. ramosa interaction, germination stimulants released into the rhizosphere are still unknown and need to be characterized.

To date, eight different classes of plant secondary metabolites, including strigolactones, isothiocyanates, sesquiterpene lactones, peagol and peagoldione, chalcones, peapolyphenols, triterpenoid saponins, or sterols, have been identified as germination stimulants for root-parasitic plants (Evidente et al. 2009, 2010, 2011; Joel et al. 2011; Virtue et al. 2006; Yoneyama et al. 2010). Among them, strigolactones are the most potent stimulants, inducing $P$. ramosa seed germination at nanomolar concentrations (Xie et al. 2007, 2008, 2009). Strigolactones constitute a new group of plant hormones involved in the regulation of shoot branching (Gomez-Roldan et al. 2008; Umehara et al. 2008). Carotenoid cleavage dioxygenase 7 (CCD7) and CCD8 are involved in the initial steps of strigolactone biosynthesis from carotenoids (Schwartz et al. 2004), and root exudates from $c c d 7$ and $c c d 8$ mutants of pea and rice show reduced ability to stimulate germination of parasitic plant seed (Gomez-Roldan et al. 2008; Umehara et al. 2008). Strigolactones have been detected in the root exudates and extracts of a wide range of monocotyledonous and dicotyledonous plants, which can be either genuine or false hosts for root-parasitic plants (Yoneyama et al. 2010). Strigolactones with diverse structures are produced by distinct host-plant species, but 5-deoxystrigol was found to be distributed widely in the plant kingdom (Xie et al. 2010). Strigolactones also act as host-recognition signals for symbiotic arbuscular mycorrhizal fungi, and phosphate starvation increases the biosynthesis and release of strigolactones in mycotrophic plants (Akiyama et al. 2005; Lopez-Raez et al. 2008; Yoneyama et al. 2007). In contrast, nonmycotrophic Brassicaceae spp. are generally thought to release little or no strigolactones into the rhizosphere; this has been demonstrated for the model plant Arabidopsis thaliana (Goldwasser et al. 2008; Kohlen et al. 2011).

$P$. ramosa germination can also be triggered by isothiocyanates, which are glucosinolate-breakdown products (Georgieva and Dimitrova-Kostova 1984, 1986; Virtue et al. 2006). Glucosinolates are thioglucoside secondary metabolites specific to the Brassicaceae, Capparidaceae, Caricaceae, and Euphorbiaceae families. Plant species synthesizing glucosinolates produce myrosinase (EC 3.2.3.1.), a $\beta$-thioglucosidase that hydrolyzes glucosinolates into a range of biologically active products, including isothiocyanates, nitriles, and oxazolidinethiones, depending on the nature of the glucosinolate, reaction conditions, and the presence of associated proteins (Bones and Rossiter 2006; Burow and Wittstock 2009). These products, and especially isothiocyanates, have a broad biocidal activity on plant pathogens and herbivores (Rask et al. 2000). In B. napus roots, myrosinase is sequestered into myrosin idioblasts located in the cortical region, and glucosinolates accumulate mainly at the root periphery. Therefore it has long been assumed that Brassicaceae plants benefit from their endogenous glucosinolate/myrosinase system only when pathogens or herbivores physically damage their tissues, thus stimulating glucosinolate hydrolysis and biocidal substance release (Bones and Rossiter 1996). However, myrosinase activity has been recorded in soils where Brassica spp. or other species grow (Al-Turki and Dick 2003; Borek et al. 1996; Gimsing et al. 2006) and concentrations of 2-phenylethyl isothiocyanate (2-PEITC), which profoundly influences the microbial community, are found in the rhizosphere of healthy, growing roots of B. napus (Rumberger and Marschner 2003). This suggests that glucosinolates released from $B$. napus roots are hydrolyzed in the rhizosphere, and that isothiocyanates could participate in the B. napus-P. ramosa interaction.

To better understand the $B$. napus $-P$. ramosa interaction, it is necessary to elucidate the exact nature and quantities of germination stimulants released into the $B$. napus rhizosphere. Here, we conducted a detailed survey of glucosinolate-breakdown products and strigolactones that accumulate in $B$. napus roots and are released into the rhizosphere to evaluate the relative contribution of these compounds to the stimulation of $P$. ramosa germination. Glucosinolate-breakdown products and strigolactones in organic solvent extracts from roots, root exudates, and field soil samples were analyzed by gas chromatography-mass spectrometry (GC-MS) and ultraperformance liquid chromatography-tandem mass spectrometry (UPLC-MS/MS), respectively (Fig. 1). Eight glucosinolate-breakdown products were identified in root extracts and two of them were detected in the $B$. napus rhizosphere. In contrast, known strigolactones were not detected in any extracts. Among glucosinolate-breakdown products, 2-PEITC was found to be the main germination stimulant of $P$. ramosa. These results, initially obtained by metabolic profiling, were further confirmed by in vitro experiments on germination stimulation activity of glucosinolatebreakdown products, co-cultivation of $B$. napus with other
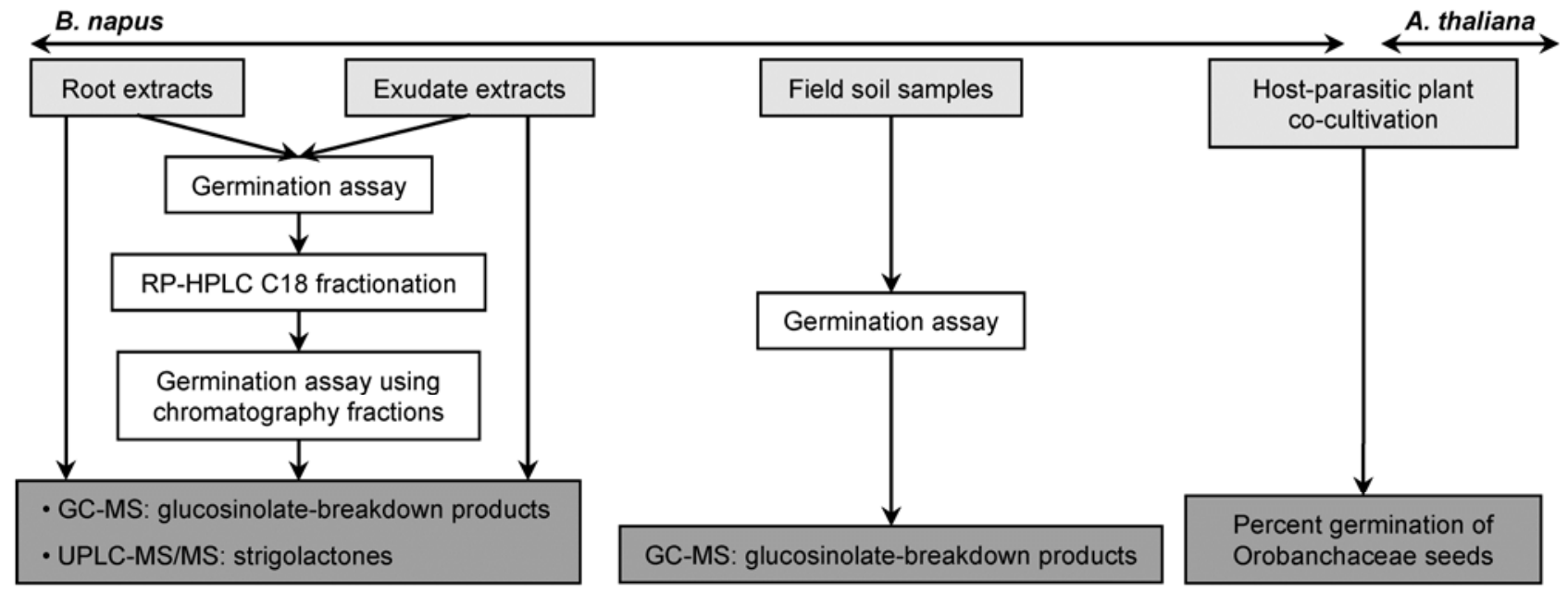

Fig. 1. Schematic diagram showing the experimental plan followed in this study to characterize germination stimulants of Phelipanche ramosa in the rhizosphere of Brassica napus. GC-MS, gas chromatography-mass spectrometry; RP-HPLC, reversed-phase high-pressure liquid chromatography; UPLC/MS-MS, ultraperformance liquid chromatography/tandem mass spectrometry. 
annual Orobanchaceae spp., and co-cultivation of Atccd7 and Atccd8 mutants with $P$. ramosa.

\section{RESULTS}

\section{Characterization and quantification}

of glucosinolate-breakdown products in B. napus roots.

Germination stimulants were extracted with ethyl acetate from roots of 6-week-old plants of B. napus 'ES Aliénor' and 'Tanto'. Each crude root extract was examined for the presence of known glucosinolate-breakdown products through a comprehensive analysis of MS fragmentation patterns and retention times (Rt) compared with those of commercial standards or from the literature. Involvement of identified compounds in the stimulation of $P$. ramosa seed germination was assessed after reversed-phase high-performance liquid chromatography (RP-HPLC) fractionation of crude root extracts followed by the determination of fraction composition and activity on seed germination (Fig. 1).

GC-MS analysis of the crude extracts revealed eight glucosinolate-breakdown products: four isothiocyanates, three nitriles, and an oxazolidinethione. The four isothiocyanates4-pentenyl isothiocyanate (peak 1), erucin (4-methylthiobutyl isothiocyanate; peak 5), 2-PEITC (peak 6), and berteroin (5methylthiopentyl isothiocyanate; peak 8)-were identified from their ion fragmentation patterns and Rt (Fig. 2A; Table 1). The nitriles 5-methylthiopentanenitrile (peak 2), 3-phenylpropanenitrile (peak 3), and 6-methylthiohexanenitrile (peak 4) and the oxazolidinethione goitrin (peak 7) were identified in a similar manner. All assignments were confirmed by cochromatography with commercial standards (data not shown), except for 5-methylthiopentanenitrile and 6-methylthiohexanenitrile, which were identified based on data from the literature (Gardiner et al. 1999). Glucosinolate-breakdown products were then quantified from the crude root extracts. Contents of isothiocyanates were slightly higher than those of nitriles, except for the relatively less abundant 4-pentenyl isothiocyanate. For instance, in ES Aliénor crude root extracts, the isothiocyanate content ranged from $1.6 \pm 1.2 \mu \mathrm{g}$ per gram of root dry weight (DW) for 4-pentenyl isothiocyanate to $38.2 \pm$ $10.1 \mu \mathrm{g}$ per gram of root DW for erucin, and nitrile content ranged from $13.8 \pm 5.5 \mu \mathrm{g}$ per gram of root DW for 5-methylthiopentanenitrile to $27.1 \pm 3.9 \mu \mathrm{g}$ per gram of root $\mathrm{DW}$ for 3-phenylpropanenitrile (Fig. 2B).

The crude root extracts from ES Aliénor stimulated germination of $79.9 \pm 2.1 \%$ of $P$. ramosa seed (Fig. 2C). The distri-

Fig. 2. Germination-stimulating activity of a root extract from 6-week-old Brassica napus plantlets ('ES Aliénor') on Phelipanche ramosa seed and associated content of glucosinolate-breakdown products determined by gas chromatography-mass spectrometry (GC-MS). A, GC-MS total ion current. Acquisition was performed using the full-scan mode. Identified glucosinolate-breakdown products are: peak 1, 4-pentenyl isothiocyanate; peak 2, 5-methylthiopentane nitrile; peak 3, 3-phenylpropanenitrile; peak 4, 6-methylthiohexane nitrile; peak 5, erucin; peak 6, 2-phenylethyl isothiocyanate (2-PEITC); peak 7, goitrin; and peak 8, berteroin. B, Quantification of glucosinolate-breakdown products after GC-MS analysis set to the selected ion monitoring mode. 3-Phenylpropanenitrile, erucin, 2-PEITC, and berteroin contents are expressed relative to the corresponding standards. 5-Methylthiopentane nitrile and 6-methylthiohexane nitrile contents are expressed in 3 -phenylpropanenitrile equivalents. Mean values \pm standard deviation (SD) are from measurements on three independent cultivations and extractions. DW = dry weight. $\mathbf{C}$, Distribution of germination-stimulating activity after reversed-phase high-performance liquid chromatography separation. The synthetic strigolactone GR24 and water were used as positive and negative controls, respectively. Mean values \pm SD are from measurements on three independent germination assays. bution of germination stimulation activity on $P$. ramosa was determined after RP-HPLC fractionation of root extracts. Germination stimulation activity was associated with fractions 11 , 12 , and 13 (11 to $14 \mathrm{~min}$ ), with fraction 12 (12 to $13 \mathrm{~min}$ ) being the most active, stimulating $61.3 \pm 8.1 \%$ germination. Then, all fractions were analyzed by GC-MS and erucin, 2-PEITC, and berteroin were identified in the active fractions. Nitriles were detected in the inactive fractions 4,5 , and 6 (4 to $7 \mathrm{~min}$ ). The corresponding fraction-eluted compound was confirmed with commercial standards (Table 2). The level of 4-pentenyl isothiocyanate in the fractions was too low for detection by GC-MS but the commercial standard of 4-pentenyl isothiocyanate eluted in active fraction 12 (Table 2). The results obtained from ES Aliénor were confirmed on Tanto.
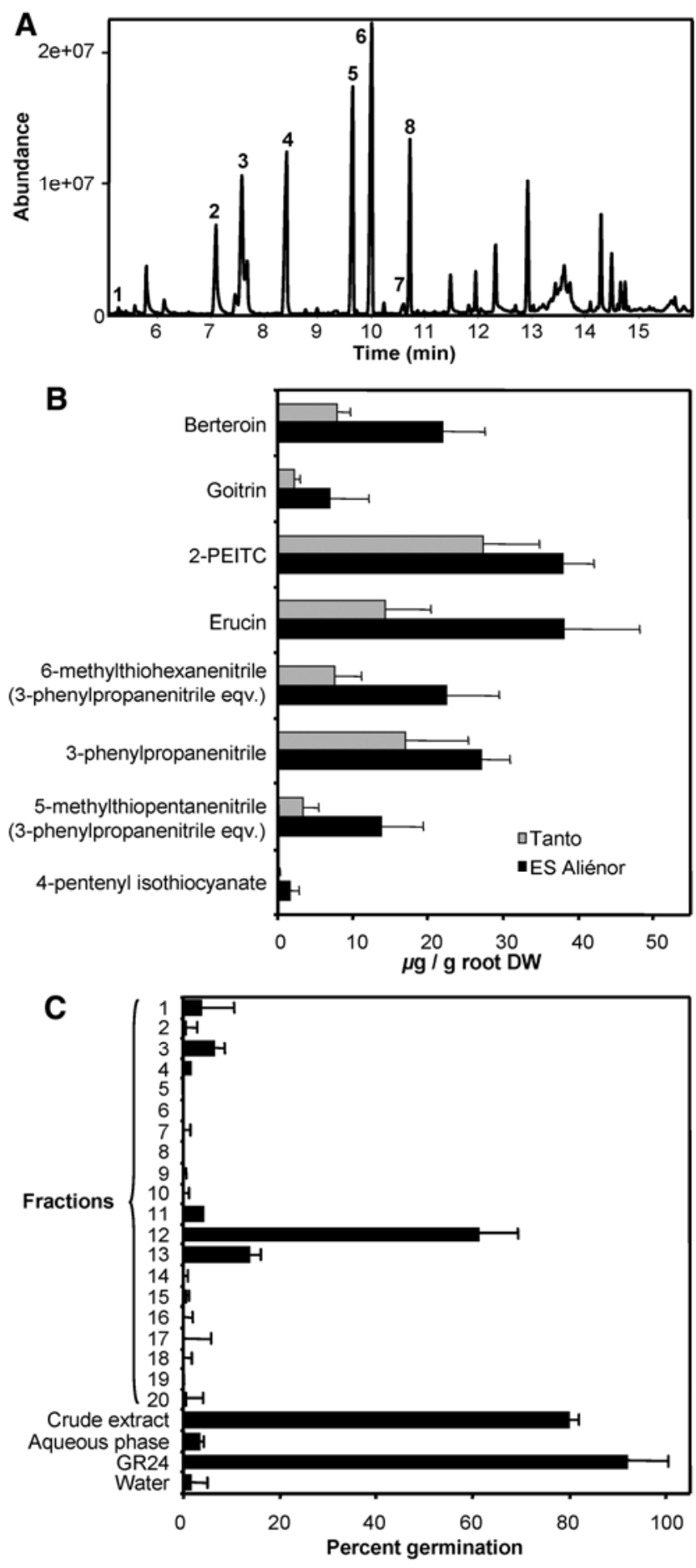
Optimization of strigolactone detection

by UPLC-MS/MS and analysis of $B$. napus root extracts.

Because strigolactone production and exudation is enhanced in mycotrophic plant species grown under phosphate starvation (Lopez-Raez et al. 2008; Yoneyama et al. 2007), we grew plantlets under phosphate deprivation to optimize detection of strigolactones in root extracts. However, because B. napus is not mycotrophic, we assumed that the strigolactone content of roots and root exudates would, nevertheless, be low. In addition, Orobanchaceae seed germination can be triggered by strigolactones at nanomolar concentrations (Kim et al. 2010) and, thus, it was necessary to optimize UPLC-MS/MS analytical conditions to reach the lowest possible limit of detection. The selected reaction monitoring (SRM) mode was chosen to identify known strigolactones by UPLC-MS/MS. Therefore, strigolactone standards $(5 \mathrm{ng} / \mu \mathrm{l})$ were first analyzed using the full-scan mode to determine their basic mass spectra. Next, product ions of the $[\mathrm{M}+\mathrm{H}]^{+}$ion, if not completely fragmented at the source level, were identified. If the $[\mathrm{M}+\mathrm{H}]^{+}$ion was entirely fragmented at the source level, we determined product ions from the source fragments of the $[\mathrm{M}+\mathrm{H}]^{+}$ion. The unfragmented $[\mathrm{M}+\mathrm{Na}]^{+}$adduct ion was used as little as possible as the precursor ion. The three transitions giving the best signal for each strigolactone were selected for further analyses (Supplementary Table 1). Some of the chosen transitions were not

Table 1. Main glucosinolate-breakdown products identified with gas chromatography-mass spectrometry in the root extracts of Brassica napus ('ES Aliénor' and 'Tanto')

\begin{tabular}{lcl}
\hline Compound identity $^{\mathbf{a}}$ & $\mathbf{R t}(\mathbf{m i n})$ & \multicolumn{1}{c}{$[\mathbf{M}-\mathbf{H}]^{-}(\mathbf{m} / \mathbf{z})^{\mathbf{b}}$} \\
\hline 4-Pentenyl isothiocyanate $\left(^{*}\right)$ & 5.68 & $127, \underline{126}, 72,70,67$ \\
5-Methylthiopentanenitrile & 7.15 & $129,82, \underline{61}$ \\
3-Phenylpropanenitrile $\left(^{*}\right)$ & 7.64 & $131, \underline{91}, \underline{65}$ \\
6-Methylthiohexanenitrile & 8.47 & $143,96,85, \underline{61}$ \\
Erucin $\left(^{*}\right)$ & 9.72 & $161, \underline{115}, 85,72,61$ \\
2-PEITC $\left(^{*}\right)$ & 10.08 & $163, \underline{91}, 65$ \\
Goitrin $(*)$ & 10.64 & $\underline{129}, 68$ \\
Berteroin $(*)$ & 10.80 & $175,129,72,68, \underline{61}$ \\
\hline
\end{tabular}

${ }^{a}$ Compound identity was confirmed by comparison of retention time (Rt) and fragmentation pattern with the commercial standard (*); 2-PEITC $=$ 2-phenylethyl isothiocyanate.

${ }^{b}$ The main fragment, underlined, was used for compound quantification, but all the ions were used for selected ion monitoring detection.

Table 2. Chromatographic behavior of standard compounds during reversedphase high-performance liquid chromatography (RP-HPLC) fractionation ${ }^{\mathrm{a}}$

\begin{tabular}{lcc}
\hline Standard identity & $\begin{array}{c}\text { Rt } \\
(\mathbf{m i n})\end{array}$ & $\begin{array}{c}\text { RP-HPLC } \\
\text { fraction }\end{array}$ \\
\hline Glucosinolate-breakdown products & & \\
3-Phenylpropanenitrile & 5.66 & $5-6$ \\
Erucin & 11.12 & 11 \\
2-PEITC & 11.93 & 12 \\
4-Pentenyl isothiocyanate & 11.93 & 12 \\
Berteroin & 12.86 & 13 \\
Goitrin & $\mathrm{ND}$ & $\mathrm{ND}$ \\
Strigolactones & & \\
7-Oxoorobanchyl acetate & 4.88 & 5 \\
Strigol & 5.35 & 5 \\
Orobanchol & 5.39 & 5 \\
2'-Epi-orobanchol & 5.43 & $5-6$ \\
GR24 & 6.38 & 6 \\
Fabacyl acetate & 9.43 & $9-10$ \\
Orobanchyl acetate & 10.65 & $10-11$ \\
Sorgolactone & 11.63 & $11-12$ \\
2'-Epi-5-deoxystrigol & 12.93 & 13 \\
5-Deoxystrigol & 13.12 & 13 \\
\hline
\end{tabular}

${ }^{\mathrm{a}} \mathrm{Rt}=$ retention time, 2-PEITC $=$ 2-phenylethyl isothiocyanate, and ND = not determined. specific to a single strigolactone standard but using the $[\mathrm{M}+\mathrm{Na}]^{+}$ion as little as possible as the precursor ion and working on source fragments of the $[\mathrm{M}+\mathrm{H}]^{+}$ion as precursor ions for transition determination dramatically lowered the limit of detection (approximately $200 \mathrm{fg}$ injected, depending on the strigolactone species), especially for 7-oxoorobanchyl acetate and sorgolactone, for which sensitivity increased 100-fold. Crude root extracts were then analyzed by UPLC-MS/MS but known strigolactones were still not detected.

Under the RP-HPLC conditions used in the separation of crude root extracts, 7-oxoorobanchyl acetate, strigol, orobanchol, and $2^{\prime}$-epi-orobanchol were eluted in fractions 5 and 6 , and fabacyl acetate in fractions 9 and 10; however, these fractions were inactive on $P$. ramosa seed germination (Table 2; Fig. 2C). Because standards of orobanchyl acetate, sorgolactone, 2'-epi-5-deoxystrigol, and 5-deoxystrigol had polarities similar to those of the isothiocyanates and were eluted in the same fractions, germination stimulation activity of these strigolactones could not be differentiated from those of isothiocyanates. After UPLC-MS/MS analysis, known strigolactones were not detected in RP-HPLC fractions.

\section{Germination stimulants released}

by $B$. napus roots in hydroponic conditions.

Isothiocyanates detected in root extracts could have been artifacts, produced from glucosinolates during root harvest due to myrosinase release from myrosin idioblasts. To ascertain isothiocyanate involvement in the $B$. napus $-P$. ramosa interaction, it was essential to identify and quantify glucosinolatebreakdown products in root exudates and in field soil samples. Furthermore, strigolactones were not identified in root extracts but these compounds may be more concentrated in root exudate extracts, because exudates were allowed to accumulate over a period of 3 days.

Therefore, plantlets ('Expert' and ES Aliénor) were grown hydroponically and root exudates released into culture medium were adsorbed on activated charcoal. Molecules adsorbed on the charcoal were eluted with acetone. After the acetone was evaporated, the residue was dissolved in small amounts of

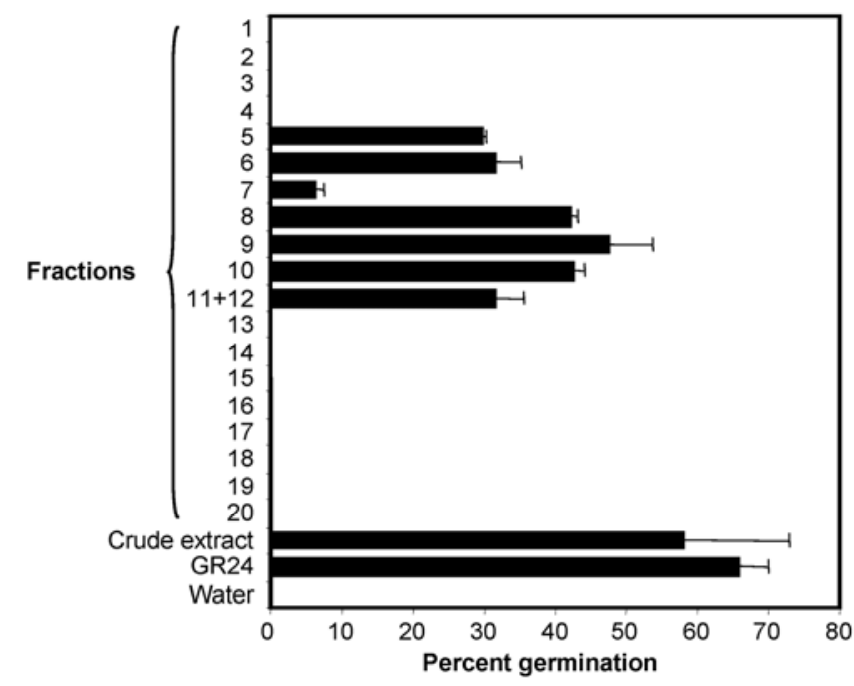

Fig. 3. Distribution of germination-stimulating activity on Phelipanche ramosa seed after reversed-phase high-performance liquid chromatography (RP-HPLC) fractionation of a root exudate extract ('Expert'). RP-HPLC fractions were 1:100 diluted to detect the less-concentrated stimulants. Fractions 11 and $12(11+12)$ were pooled and concentrated twofold before the germination assay. GR24 and water were used as positive and negative controls, respectively. Mean values \pm standard deviation are from measurements on three independent germination assays. 
water and extracted with ethyl acetate to obtain a crude extract (Fig. 1). This crude extract triggered seed germination of $P$. ramosa (Fig. 3). It was then separated by RP-HPLC and seven active fractions were identified. Fractions 5 and 6 corresponded to the Rt of 7-oxoorobanchyl acetate, orobanchol, and its isomers, and fractions 9 and 10 to fabacyl acetate and orobanchyl acetate (Table 2). However, using UPLC-MS/MS, these strigolactones were not identified in the crude extract or in the fractions obtained from the exudate. Conversely, 2-PEITC was identified using GC-MS in the crude extract as well as in exudate fractions 11 and 12. For Expert, 2-PEITC production in root exudates was $3.9 \mathrm{ng} / \mathrm{day} / 100$ plants. 3-Phenylpropanenitrile was also present in the crude extract and in fractions 5 and 6. Other glucosinolate-breakdown products were not detected. These results obtained from Expert were confirmed on ES Aliénor.

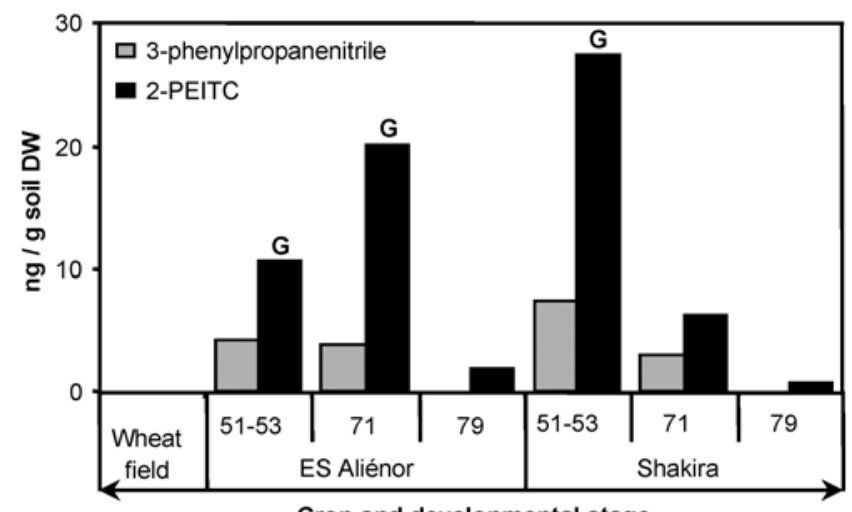

Crop and developmental stage

Fig. 4. Quantification of glucosinolate-breakdown products in field soil samples from the rhizosphere of 'ES Aliénor' and 'Shakira'. After extraction from the soil, compounds were quantified by gas chromatographymass spectrometry analysis set to the selected ion monitoring mode. 3Phenylpropanenitrile and 2-phenylethyl isothiocyanate (2-PEITC) content are expressed relative to their corresponding standards. Brassica napus developmental stages were determined following the international $\mathrm{BBCH}$ scale: 51 to 53 , attached buds stage; $71,10 \%$ pods displayed their final size; 79, all the pods reached their final size (Lancashire et al. 1991). DW = dry weight and G above bars = Phelipanche ramosa seed germination induced by the extract.
Germination stimulants in B. napus field soil samples.

Glucosinolate-breakdown products were extracted from infested field soil samples of B. napus ES Aliénor and 'Shakira' with dichloromethane and extracts were analyzed using GCMS (Fig. 1). Field soil samples from a neighboring wheat field were used as a negative control. As in root exudates of hydroponically grown plantlets, 3-phenylpropanenitrile and 2-PEITC were the only glucosinolate-breakdown products identified in the $B$. napus rhizosphere, at the $\mathrm{BBCH}-51$ to $\mathrm{BBCH}-53$ and $\mathrm{BBCH}-71$ phenological stages. At the $\mathrm{BBCH}-79$ stage, only 2-PEITC was identified in both $B$. napus cultivars. As observed in roots, the amount of 2-PEITC was greater than that of 3phenylpropanenitrile. Its content reached $20.1 \mathrm{ng}$ per gram of soil DW at the BBCH-71 stage for ES Aliénor and $27.5 \mathrm{ng}$ per gram of soil DW at the BBCH-51 to BBCH-53 stage for Shakira (Fig. 4). The highest observed 3-phenylpropanenitrile content was 4.3 ng per gram of soil DW for ES Aliénor and 7.4 ng per gram of soil DW for Shakira at the BBCH-51 to BBCH-53 stage (Fig. 4). Glucosinolate-breakdown products were not identified in field soil samples from the neighboring wheat plot, indicating that they must be released by $B$. napus. Dichloromethane extracts containing the highest levels of 2-PEITC (ES Aliénor during the BBCH-51 to BBCH-53 and BBCH-71 developmental stages and Shakira during the BBCH-51 to BBCH-53 stage) stimulated P. ramosa seed germination. Taken together, these results demonstrated that 2PEITC, a potent germination stimulant of $P$. ramosa, was present in the B. napus rhizosphere of an infested field.

\section{Determination of compound activities in the stimulation of $P$. ramosa seed germination using in vitro bioassays.}

A set of five strigolactones (GR24, strigol, 7-oxoorobanchyl acetate, fabacyl acetate, and orobanchol) and six isothiocyanates (2-PEITC, 4-pentenyl isothiocyanate, benzyl isothiocyanate, sulforaphane [4-methylsulfinylbutyl isothiocyanate], berteroin, and erucin) was tested in vitro on $P$. ramosa germination. Strigolactones and isothiocyanates induced maximums of more than $90 \%$ seed germination. Strigolactones induced $P$. ramosa seed germination with half maximal effective concentration $\left(\mathrm{EC}_{50}\right)$ values in the picomolar range (from $2.5 \times 10^{-10} \pm$ $6.0 \times 10^{-11} \mathrm{M}$ for fabacyl acetate to $2.0 \times 10^{-12} \pm 1.1 \times 10^{-12} \mathrm{M}$ for orobanchol), and isothiocyanate $\mathrm{EC}_{50}$ values were in the
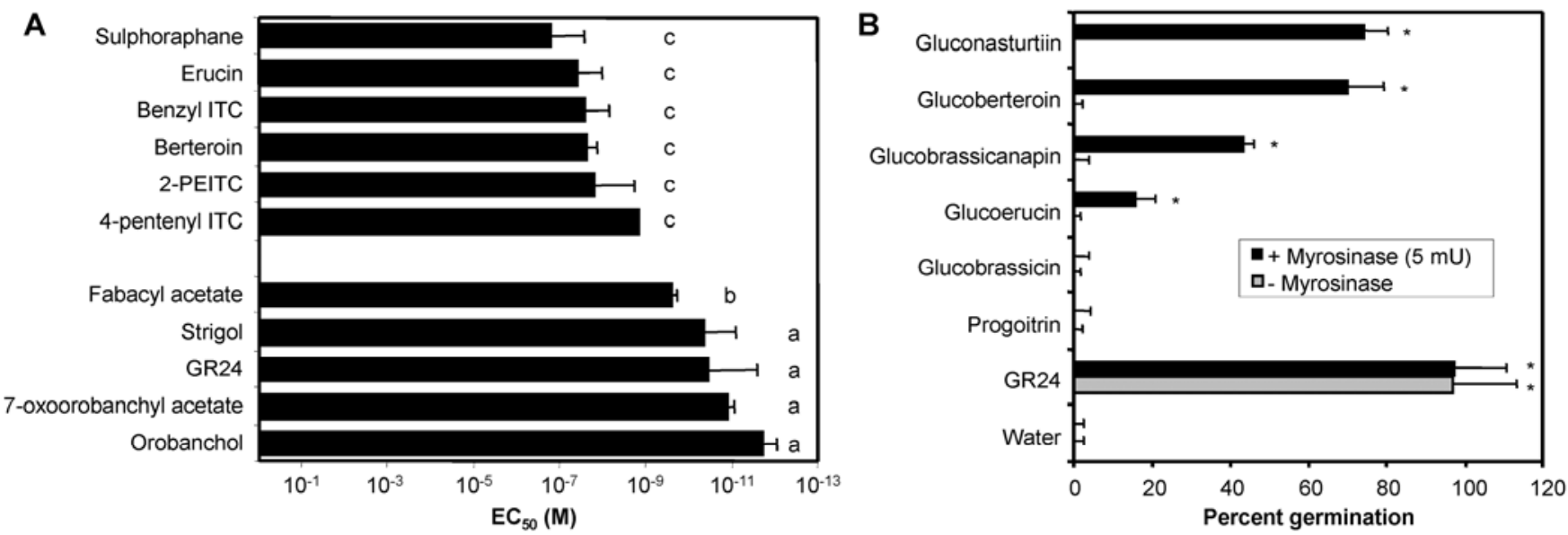

Fig. 5. Structure-activity relationship of standard compounds on Phelipanche ramosa seed germination. A, Concentration-dependent stimulation by isothiocyanates (2-phenylethyl isothiocyanate [2-PEITC], 4-pentenyl isothiocyanate [ITC], benzyl ITC, sulforaphane, berteroin, and erucin) or strigolactones (GR24, strigol, 7-oxoorobanchyl acetate, fabacyl acetate, and orobanchol). $\mathrm{EC}_{50}=$ half maximal effective concentration. Mean values \pm standard error are from measurements on at least three independent dilution ranges, analyzed by three independent germination assays. Values with the same letter are not significantly different (analysis of variance [ANOVA], Student-Newman- Keuls test, $P<0.05)$. B, Stimulating activity of glucosinolates $\left(10^{-7}\right.$ M) in the presence or absence of $5 \mathrm{mU}$ myrosinase. GR24 and water were used as positive and negative controls, respectively. Mean values \pm standard deviation are from measurements on three independent germination assays. An asterisk (*) indicates that values are significantly different between the treatment and the water control without myrosinase (ANOVA and Holm-Sidak multiple comparison test, $P<0.05$ ). 
nanomolar range (from $1.7 \times 10^{-7} \pm 1.4 \times 10^{-7} \mathrm{M}$ for sulforaphane to $1.5 \times 10^{-9} \pm 1.0 \times 10^{-11} \mathrm{M}$ for 4-pentenyl isothiocyanate) (Fig. 5A). In contrast, the other glucosinolate-breakdown products such as 3-phenylpropanenitrile, goitrin, and indole-3-carbinol were inactive in the concentration range tested $\left(10^{-5}\right.$ to $\left.10^{-17} \mathrm{M}\right)$.

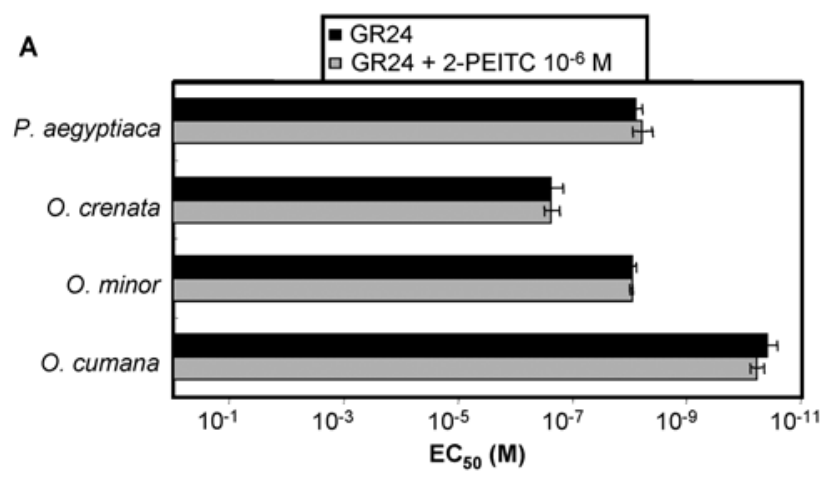

B

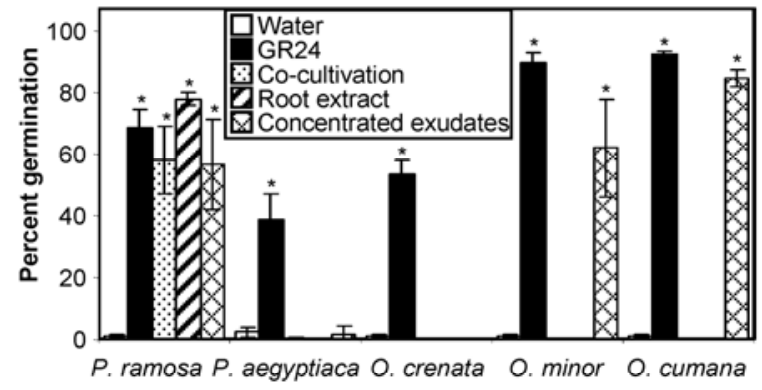

C

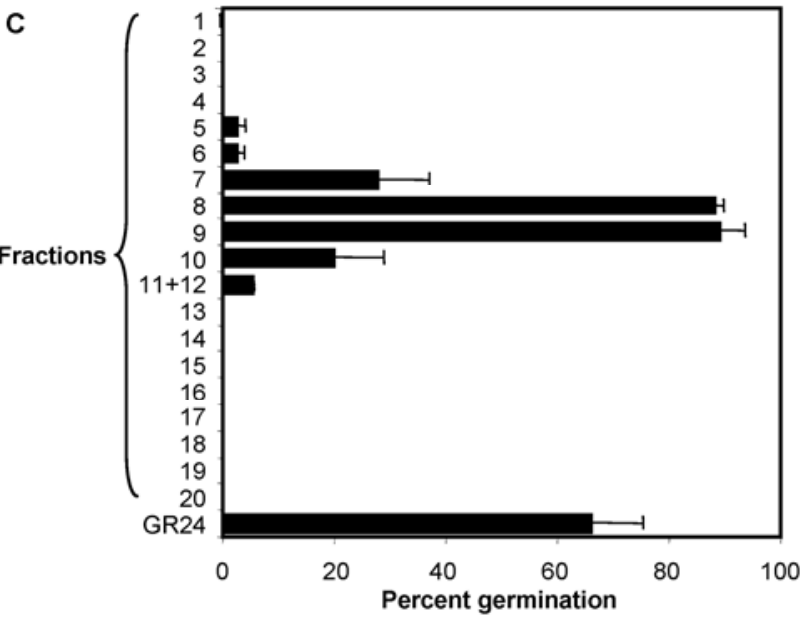

Fig. 6. Germination of other Orobanchaceae spp. in the presence of 2 phenylethyl isothiocyanate (2-PEITC) or germination stimulants from 3week-old Brassica napus plantlets. A, Half maximal effective concentration $\left(\mathrm{EC}_{50}\right)$ of GR24 in the presence or absence of $10^{-6} \mathrm{M}$ 2-PEITC. B, Seed germination in B. napus rhizosphere during co-cultivation experiments (dotted bars) or after in vitro stimulation with root extracts ('ES Aliénor') (hatched bars) and concentrated root exudates extract ('Expert', 1:100 dilution) (cross-hatched bars). Water (white bars) and GR24 (black bars) were used as negative and positive controls, respectively. An asterisk (*) indicates that values are significantly different between the species-treatment and the water control (ANOVA and HolmSidak multiple comparison test, $P<0.05$ ). C, Distribution of germination-stimulating activity on Orobanche cumana seed of a root exudate extract (Expert) fractionated by reversed-phase high-performance liquid chromatography (RP-HPLC). RP-HPLC fractions were 1:100 diluted. Fractions 11 and $12(11+12)$ were pooled and concentrated twofold before the germination assay. GR24 was used as the positive control. Bars represent means \pm standard deviation (SD) of five independent plates for the co-cultivation experiment or means \pm SD of three independent germination assays.
To determine the effect of glucosinolate, a set of six compounds (gluconasturtiin, glucoerucin, glucoberteroin, glucobrassicanapin, progoitrin, and glucobrassicin) was chosen from glucosinolate-breakdown products identified in the present study and in the literature (Gardiner et al. 1999; Kirkegaard and Sarwar 1998). In the concentration range tested $\left(10^{-5}\right.$ to $10^{-17} \mathrm{M}$ ), these six glucosinolates were inactive (data not shown). By adding $5 \mathrm{mU}$ of myrosinase to the germination media containing a glucosinolate (at a final concentration of $\left.10^{-7} \mathrm{M}\right)$, germination was significantly $(P<0.05)$ stimulated in the case of gluconasturtiin, glucoberteroin, glucobrassicanapin, and glucoerucin, whereas no seed germinated in the presence of glucobrassicin or progoitrin (Fig. 5B). This result corroborates the identification of isothiocyanates as germinationstimulating compounds. Gluconasturtiin, glucoberteroin, glucobrassicanapin, and glucoerucin are hydrolyzed by myrosinase into stable isothiocyanates (2-PEITC, berteroin, 4-pentenyl isothiocyanate, and erucin, respectively), whereas hydrolysis of glucobrassicin and progoitrin leads to indole-3-carbinol (and various other compounds) and to goitrin, respectively (Agerbirk et al. 2009; Kirkegaard and Sarwar 1998).

\section{2-PEITC and $B$. napus root exudate activity} on other parasitic plant seed.

The germination stimulation capacity of 2-PEITC was investigated in vitro on $O$. minor, O. cumana, O. crenata, and $P$. aegyptiaca populations. Germination ability of seed batches was checked beforehand with the determination of the $\mathrm{EC}_{50}$ of GR24, which ranged from $4.2 \times 10^{-11} \pm 1.5 \times 10^{-11} \mathrm{M}$ for $O$. cumana to $2.5 \times 10^{-7} \pm 1.1 \times 10^{-7}$ for $O$. crenata (Fig. $6 \mathrm{~A}$ ). 2 PEITC at $10^{-5} \mathrm{M}$ to $10^{-11} \mathrm{M}$ did not stimulate seed germination of $O$. minor, O. cumana, or $O$. crenata but induced $35.7 \pm$ $12.9 \% \quad P$. aegyptiaca seed germination at $10^{-5} \mathrm{M}$ (data not shown). Therefore, 2-PEITC effect was almost specific to $P$. ramosa compared with the other Orobanchaceae spp. tested. In addition, $10^{-6} \mathrm{M}$ 2-PEITC did not significantly modify the $\mathrm{EC}_{50}$ of GR24 for $O$. minor, O. cumana, O. crenata, or P. aegyptiaca $(P<0.05)$, indicating that 2-PEITC did not interfere with seed germination of these species after stimulation by GR24 (Fig. 6A).

To determine whether other stimulants from B. napus (ES Aliénor) root exudates could induce seed germination of the various Orobanchaceae spp., co-cultivation experiments were carried out (Fig. 1). After 2 weeks, B. napus root exudates induced the germination of $P$. ramosa seed only $(59.6 \pm 11.2 \%)$. $O$. minor, O. cumana, O. crenata, and $P$. aegyptiaca only showed negligible germination rates (less than $1 \%$ ), which corresponded to spontaneous germination of sterilized and preconditioned seed (between 0 and 2\%) $(P<0.05)$ (Fig. 6B). The lack of any root exudate effect was supported by the absence of seed germination in the presence of root extracts (Fig. 6B). One possible explanation is that other stimulants, including strigolactones, were not present in high enough concentrations to trigger seed germination of these Orobanchaceae spp., which detect strigolactones. Alternatively, B. napus roots may release inhibitors of $O$. minor, O. cumana, O. crenata, and $P$. aegyptiaca seed germination. However, when root exudates were concentrated during hydroponic culture of $100 \mathrm{~B}$. napus plants, the extracts triggered not only $P$. ramosa but also $O$. minor and $O$. cumana seed germination $(P<0.05)$ (Fig. 6B). This result supports the hypothesis that, in the B. napus rhizosphere, germination stimulants of at least $O$. minor and $O$. cumana were not concentrated enough to trigger seed germination. When RP-HPLC fractions were diluted 1:100 for the germination assay, germination stimulation was observed only for $O$. cumana. The major germination-stimulating activity was detected in fractions 7 to 10 (Fig. 6C). 
Contribution of strigolactones and isothiocyanates to the A. thaliana-P. ramosa interaction.

Given that $C C D 7$ and $C C D 8$ are involved in strigolactone biosynthesis (Mashiguchi et al. 2009), we used the corresponding A. thaliana mutants (max3-9 and max4-1 alleles) (Booker et al. 2004; Sorefan et al. 2003) for co-cultivation experiments with $P$. ramosa to screen for parasitic seed germination defects. We chose to work on $A$. thaliana mutants because this species produces both strigolactones and glucosinolates (Brown et al. 2003; Goldwasser et al. 2008; Kohlen et al. 2011). The percentage of germination of $P$. ramosa seed exposed to the $\max 3-9$ root system was not significantly different ( $t$ test $P<0.05$ ) from those exposed to the ecotype Columbia- 0 $($ Col-0) wild-type roots $(56.2 \pm 18.2$ versus $64.9 \pm 17.5 \%)$ (Fig. 7). Similar results were obtained for $\max 4-1$ plants $(63.8 \pm$ $18.4 \%$ ) (Fig. 7). This result clearly demonstrates that the biosynthesis of the major germination stimulants for $P$. ramosa produced by $A$. thaliana was not affected in these strigolactone mutants.

\section{DISCUSSION}

\section{Glucosinolate-breakdown products were identified} in B. napus roots and its rhizosphere.

Identification of germination stimulants released from $B$. $n a$ pus into the rhizosphere is a key step toward the understanding of the interaction between $B$. napus and P. ramosa. Assuming that glucosinolate-breakdown products are involved in the host-parasite interaction, we set out to determine the nature of the breakdown products that accumulate in B. napus roots and are released into the rhizosphere. Our results indicate that gluconasturtiin or 3-phenylpropanenitrile and 2-PEITC are released from $B$. napus roots into the rhizosphere, whereas glucoerucin and glucoberteroin only accumulate in but are not released from roots. Indeed, 2-PEITC, erucin, berteroin, and 4-pentenyl isothiocyanate as well as three nitriles and goitrin were identified and quantified in root extracts. Each nitrile shares a parent glucosinolate with one of the characterized isothiocyanates. For instance, 2-PEITC and 3-phenylpropanenitrile are both breakdown products of gluconasturtiin, the most abundant glucosinolate in B. napus roots (Kirkegaard and Sarwar 1998). 5-Methylthiopentanenitrile and erucin are derived from glucoerucin, and 6-methylthiohexanenitrile and berteroin are derived from glucoberteroin. 4-Pentenyl isothiocyanate and goitrin are derived from glucobrassicanapin and progoitrin, respectively (Kirkegaard and Sarwar 1998). Therefore, parent glucosinolates of all the breakdown products characterized in this study are root glucosinolates previously described in the literature (Gardiner et al. 1999; Kirkegaard and Sarwar 1998). Interestingly, relatively high contents of glucoerucin-, gluconasturtiin-, and glucoberteroin-derived nitriles or isothiocyanates were detected in root extracts but only the gluconasturtiin-derived compounds were present in field soil samples and in root exudates. The levels of breakdown products in the rhizosphere seem to be related to the amounts of released glucosinolates, because the degradation kinetics of glucosinolates is independent of glucosinolate structure (Gimsing et al. 2006).

\section{2-PEITC is the main stimulant}

\section{of $P$. ramosa seed germination in the $B$. napus rhizosphere.}

In this work, we demonstrated that seed germination of a $P$. ramosa population virulent on $B$. napus was triggered by isothiocyanates, as characterized by RP-HPLC separation, germination assays, and GC-MS analyses. In effect, 2-PEITC was the only isothiocyanate identified in the B. napus rhizosphere and appeared to be the major glucosinolate-breakdown product involved in the B. napus-P. ramosa interaction. This is the first report of an isothiocyanate involved in P. ramosa seed germination in the $B$. napus rhizosphere. Although previously suggested, germination stimulants released from $B$. napus roots have never been identified before (Georgieva and DimitrovaKostova 1983, 1986; Virtue et al. 2006). In our study, among the glucosinolates and the glucosinolate-breakdown products examined, $P$. ramosa germination was induced only by isothiocyanates. These results should be interpreted with caution because germination tests using standard compounds may not reflect the full germination stimulation spectrum generated by naturally occurring glucosinolates, which probably involves a mixture of various breakdown products. However, we did not observe any germination stimulation by compounds other than isothiocyanates (glucosinolates, nitriles, goitrin, and indole-3carbinol). Moreover, identification of isothiocyanates as germination-stimulating compounds was demonstrated in a germination assay with glucosinolates, because glucosinolates stimulated $P$. ramosa seed germination only after myrosinase hydrolysis into stable isothiocyanates. Otherwise, in root extracts, isothiocyanates (4-pentenyl isothiocyanate, erucin, 2PEITC, and berteroin) were the only germination stimulants identified by MS, and RP-HPLC fractions stimulating seed germination contained only isothiocyanates.

In the $B$. napus rhizosphere, $P$. ramosa was the only Orobanchaceae sp. that germinated during co-cultivation experiments. We demonstrated that seed of other Orobanchaceae spp. did not respond to a biological concentration of 2-PEITC whereas they responded to GR24. These results support the conclusion that $P$. ramosa germination was triggered by 2 -PEITC released from $B$. napus roots. Our results also ruled out the possibility of germination inhibitors in the B. napus rhizosphere for $O$. minor and $O$. cumana, although they were not ruled out for $P$. aegyptiaca and $O$. crenata. Moreover, the $\mathrm{EC}_{50}$ values obtained for isothiocyanates were in accordance with the concentrations determined in the B. napus rhizosphere. Indeed, the maximal content of 2-PEITC recorded in field soil samples by Rumberger and Marschner (2003) was almost 2,000 pmol per gram of soil. This is in agreement with our results on field soil extracts, which were of the same order, and this content must be sufficient to induce $P$. ramosa seed germination, the $\mathrm{EC}_{50}$ of 2-PEITC being $1.66 \times 10^{-8} \mathrm{M}$ (i.e., $16.6 \mathrm{pmol} / \mathrm{ml}$ ).

However, when we used highly concentrated root exudates from hydroponic culture experiments with many $B$. napus plants, germination stimulation activity on $P$. ramosa was detected in fractions 5 and 6 and 7 to 10 of RP-HPLC, and fractions 7 to 10 also stimulated $O$. cumana germination. These stimulants may be strigolactones, because the standards of

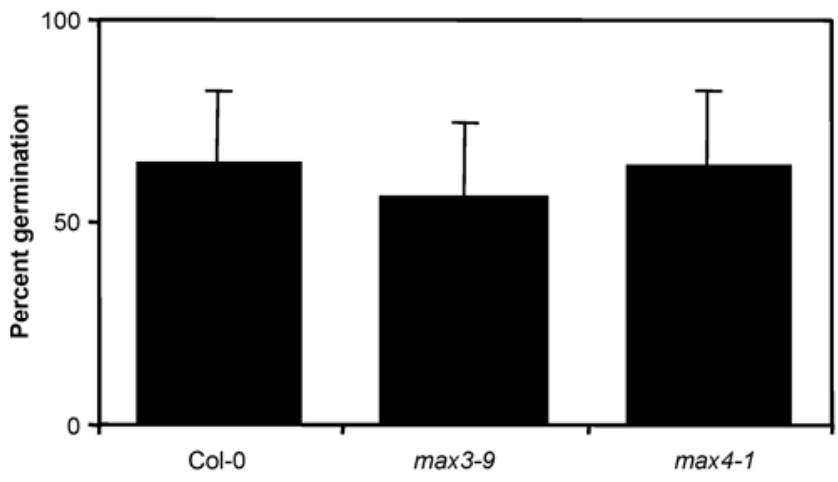

Fig. 7. Percentage of Phelipanche ramosa seed germinated in the rhizosphere of the Arabidopsis thaliana strigolactone mutants Atmax3-9 and Atmax4-1 and compared with the Col-0 ecotype. Attached P. ramosa plantlets were counted as germinated seed. Mean values \pm standard deviation resulted from measurements on 10 independent plates and were compared using a $t$ test $(P<0.05)$. 
7-oxoorobanchyl acetate and orobanchol and its isomers eluted in fractions 5 and 6 and those of fabacyl acetate and orobanchyl acetate in fractions 9 and 10. However, UPLCMS/MS analyses did not identify these strigolactones in either the RP-HPLC fractions or the crude extracts. Therefore, if these germination stimulants are known strigolactones, their content in exudate extracts was below the UPLC-MS/MS limit of detection (200 fg/injection) and below the $O$. minor sensitivity limit $\left(10^{-10} \mathrm{M}\right.$ and greater, depending on the strigolactone species) (Kim et al. 2010) in the fractions at a 1:100 dilution. However, none of our strigolactone standards had an Rt corresponding to fractions 7 and 8 . Therefore, we assume that the stimulants present in fractions 7 and 8 are stillunknown strigolactones or belong to another family of compounds, which trigger $P$. ramosa and $O$. cumana germination.

In the field, 2-PEITC appears to be the main germination stimulant in the B. napus rhizosphere, and strigolactones or other germination stimulants may play other roles in the chemical communication between the host and the parasite. In $B$. napus roots with secondary growth, the highest glucosinolate concentrations are observed just under the outermost periderm layer, which promotes the release of glucosinolates in the rhizosphere during root expansion (McCully et al. 2008). Localization of myrosin idioblasts in the innermost tissues of the root suggests that isothiocyanates themselves are not released from the roots and that glucosinolates are hydrolyzed by myrosinase at the root surface or in the rhizosphere by microorganisms expressing myrosinase (Cheng et al. 2004; Gimsing et al. 2006; McCully et al. 2008; Sakorn et al. 2002). This could explain why the 2-PEITC content was so low in root exudate extracts, which were obtained from plants cultivated in controlled conditions. Severe root injury by a root pathogen may also activate glucosinolate breakdown by B. napus myrosinase. B. napus-P. ramosa appears to be a complex pathosystem: their interaction may also depend on the presence of myrosinase-producing microorganisms in the vicinity of $B$. napus roots or potential root-damaging organisms. These potential additional players in the pathosystem constitute an original finding, which suggests that soil micro- or macroflora are involved in the parasitism of B. napus by $P$. ramosa.

\section{Isothiocyanates can also be involved \\ in the A. thaliana-P. ramosa interaction.}

Isothiocyanates may be involved in other host-parasite interactions, such as that between the model plant A. thaliana and $P$. ramosa. With co-cultivation experiments, we demonstrated that the percentage of $P$. ramosa germination in the rhizosphere of strigolactone-deficient mutants Atmax3-9 and Atmax4-1 was not significantly different from that in the rhizosphere of the wild-type Col-0, indicating that these mutants did not lack their principal germination stimulants. Glucoraphanin, the precursor of sulforaphane, is the main isothiocyanate-producing glucosinolate accumulated in A. thaliana roots of the Col-0 ecotype (Brown et al. 2003). Therefore, sulforaphane, which stimulated $P$. ramosa seed germination with an $\mathrm{EC}_{50}$ value of $1.7 \times 10^{-7} \mathrm{M}$ in our study, may be involved in the interaction between A. thaliana and P. ramosa. Given that, in our RP-HPLC conditions, the Rt of relatively non-polar strigolactones, including orobanchyl acetate, sorgolactone, 2'epi-5-deoxystrigol, and 5-deoxystrigol, were similar to those of isothiocyanates, sulforaphane may even correspond to one of the unknown germination stimulants identified in A. thaliana root exudates (Goldwasser et al. 2008; Kohlen et al. 2011).

Therefore, in A. thaliana, two families of germination stimulants, isothiocyanates and strigolactones, may be involved in the induction of $P$. ramosa seed germination. This finding is in accordance with previous studies, concluding that Brassicaceae plants may release different classes of germination stimulants (Fernández-Aparicio et al. 2009; Goldwasser and Yoder 2001). In spite of extensive studies, cultivars or mutants that have completely lost their ability to induce parasitic plant seed germination have not been found in A. thaliana (Goldwasser and Yoder 2001; Westwood 2000). Otherwise, if only strigolactones were involved in germination stimulation, poor induction of germination would be expected with plant exudates of nonmycotrophic plant species, such as A. thaliana. In contrast, $A$. thaliana root exudates actively stimulate germination of Phelipanche spp. (Goldwasser et al. 2008; Kohlen et al. 2011).

\section{Isothiocyanate recognition and evolution of $P$. ramosa.}

Among the $P$. ramosa populations that can be found worldwide, isothiocyanates stimulate at least the germination of the most virulent population on B. napus in France. Although other weedy Orobanchaceae spp. such as $O$. minor, O. cumana, and $O$. crenata were considered here, isothiocyanate activity was specific to $P$. ramosa and, to a lesser extent, $P$. aegyptiaca. This finding is in accordance with the hypothesis of a selective pressure imposed by a new host, B. napus, favoring the emergence of a virulent $P$. ramosa population (Benharrat et al. 2005; Brault et al. 2007). It has already been demonstrated that host-driven selection pressures have played an important role in the evolutionary diversification of the parasitic angiosperms (de Vega et al. 2008; Thorogood et al. 2008, 2009). Recent evolution in the response to particular stimulants from crops has already been reported. For instance, O. minor var. minor germination is induced by GR24 while $O$. minor subsp. maritima is unresponsive to it, and Scorpiurus muricatus, a host of non-weedy $O$. foetida populations, does not stimulate seed germination of weedy $O$. foetida populations (Pujadas-Salva 1999; Thorogood et al. 2009). This evolutionary diversification can partly explain the broad effect of some germination stimulants such as strigolactones and trans-22-dehydrocampesterol, whereas isothiocyanates, dehydrocostus lactone, peagol and peagoldione, chalcones, peapolyphenol A, and soyasapogenol B have a more specific effect (Evidente et al. 2009, 2010, 2011; Joel et al. 2011).

Along these lines, dehydrocostuslactone, a sesquiterpene lactone, was recently identified as the main germination stimulant of O. cumana in the sunflower (Helianthus annuus) rhizosphere (Joel et al. 2011). Isothiocyanates and sesquiterpene lactones are secondary metabolites characteristic of the Brassicaceae and Asteraceae families, respectively, and function in chemical defense and plant-insect signaling (Mullin et al. 1991; Seaman 1982; Spring et al. 1982). Similar to isothiocyanates, which only stimulate $P$. ramosa seed germination, dehydrocostuslactone specifically triggered $O$. cumana germination of the various species tested, giving another example of evolution of the seed response to germination stimulants, which may have been driven by host selective pressure (Joel et al. 2011). The identification of new classes of germination stimulants, structurally different from strigolactones but exhibiting high activities, demonstrates the large adaptation potential of Orobanchaceae spp.

In conclusion, basic research is required to decipher the molecular events involved in $P$. ramosa germination after stimulation by strigolactones or isothiocyanates and to study the $P$. $r a-$ mosa response to isothiocyanates in $P$. ramosa populations that have not evolved in contact with Brassicaceae spp. Understanding the relative contribution of isothiocyanates and other germination stimulants in $B$. napus $-P$. ramosa interaction under field conditions will help guide breeders in their breeding programs for resistant B. napus lines. 


\section{MATERIALS AND METHODS}

Plant material.

P. ramosa seed were collected from mature flowering spikes in an oilseed rape field at Saint-Martin-de-Fraigneau, France in 2007. P. aegyptiaca seed were collected in a tomato crop in Israel in 2011; O. minor in a red clover field in Utsunomiya, Japan in 2009; O. cumana in a sunflower field in Longevillesur-Mer, France in 2009; and $O$. crenata in a pea field in Ariana, Tunisia in 2007. All seed populations were stored in the dark at $25^{\circ} \mathrm{C}$ until use.

The $B$. napus cultivars all displayed low-erucic acid and glucosinolate content in seed (00-type). Tanto (spring type), ES Aliénor, and Expert (winter type) were chosen for biochemical analysis because they are highly susceptible to $P$. ramosa. They were obtained from Institut National de la Recherche Agronomique (INRA) (Le Rheu, France), EURALIS Semences (Lescar, France), and SARL Adrien Momont \& Fils (Mons-en-Pévèle, France), respectively. Glucosinolate-breakdown products were also extracted from the rhizosphere of Shakira (winter type; Maïsadour Semences, Mont-de-Marsan, France) infested by $P$. ramosa. ES Aliénor was chosen for cocultivation experiments.

A. thaliana wild-type plants and the max mutants had the genetic background of Col-0. The max alleles were obtained from the European Arabidopsis Stock Center (NASC). One representative mutant allele was used per gene: $\operatorname{max3}$-9 for AtCCD7 (NASC ID: N9567) and max4-1 for AtCCD8 (NASC ID: N9568).

\section{Chemicals and reagents for biochemical analysis.}

Commercial standards of glucosinolate-breakdown products were benzyl isothiocyanate, 3-phenylpropanenitrile, 2-PEITC, sulforaphane (Sigma-Aldrich, St. Louis), erucin, berteroin (LKT Laboratories, St. Paul, MN, U.S.A.), DL-goitrin, and indole-3-carbinol (VWR, West Chester, PA, U.S.A.). Phenanthrene was purchased from Sigma-Aldrich. Each stock solution was made up to $10^{-1} \mathrm{M}$ by diluting standards in an appropriate volume of acetone for germination assays and was prepared at $5 \mu \mathrm{g} / \mu \mathrm{l}$ in hexane for GC-MS analysis. Working solutions were prepared by diluting stock solutions in acetone or in hexane. Glucosinolates were from Phytoplan (Diehm and Neuberger GmbH, Heidelberg, Germany). Stock solutions were prepared at $1 \mathrm{mg} / \mathrm{ml}$ by diluting powders in water and working solutions were dilutions in water. $2^{\prime}$-Epi-orobanchol, orobanchyl acetate, 5-deoxystrigol, 2'-epi-5-deoxystrigol, strigol, orobanchol, fabacyl acetate, and 7-oxoorobanchyl acetate were purified at Utsunomiya University (Japan). GR24 (a synthetic strigolactone) and sorgolactone were a gift from F. D. Boyer (Centre National de la Recherche Scientifique [CNRS]-INRA Gif-sur-Yvette, France). Stock solutions were prepared at $250 \mathrm{ng} / \mu \mathrm{l}$ in acetonitrile. Working solutions were dilutions in water for germination assays and in acetonitrilewater-acetic acid, 50:50:0.1 ( $\mathrm{vol} / \mathrm{vol} / \mathrm{vol})$ for UPLC-MS/MS analyses. All solutions were stored at $-20^{\circ} \mathrm{C}$.

Acetic acid, acetonitrile, dichloromethane, ethyl acetate, and anhydrous hexane were HPLC-grade quality (Sigma-Aldrich). Water was purified using a Milli-Q system (Purelab Option-Q; Elga Labwater, High Wycombe, U.K.). HEPES, $\mathrm{K}_{2} \mathrm{HPO}_{4}$, and anhydrous $\mathrm{MgSO}_{4}$ were purchased from Sigma-Aldrich.

\section{Plant growth conditions and material collection for biochemical analysis.}

B. napus seed were surface sterilized with $12 \%$ sodium hypochlorite and thoroughly rinsed with distilled water. Before sampling $B$. napus roots, plants were grown under sterile conditions. Plastic containers ( 40 by 30 by $21 \mathrm{~cm}$, width by length by height $[\mathrm{W} \times \mathrm{L} \times \mathrm{H}]$ ) equipped with a faucet and filled with $3 \mathrm{~cm}$ of gravel under $4 \mathrm{~cm}$ of sand were autoclaved. Fifty seed were sown per container in moistened sand, under laminar air flow protection. To maintain sterile conditions during plant growth, containers were covered by $50 \mathrm{~cm}$ of overhanging transparent plastic bags. Three 50-plant containers were used for each accession. They were placed in a growth room (21 and $17^{\circ} \mathrm{C}$, day and night, respectively, for a 16 -h photoperiod, at $70 \%$ humidity) and were watered with filtered (MILLEX GP filter, 0.22- $\mu \mathrm{m}$ polyethersulfone [PES] membrane) (Millipore, Carrigtwohill, Ireland) $0.5 \times$ Tadano and Tanaka growth medium containing $16 \mu \mathrm{M} \mathrm{Na} \mathrm{NPO}_{4}$ (Tadano and Tanaka 1980). The roots were constantly oxygenated by $0.2-\mu \mathrm{m}$-filtered bubbling. Five weeks after sowing, the growth medium was substituted with distilled water. Plants were removed after 6 weeks; roots were frozen in liquid nitrogen and stored at $-80^{\circ} \mathrm{C}$ before extraction of germination stimulants.

For the hydroponic growth of B. napus plantlets, seed ('Expert' and 'ES Aliénor') were germinated on moistened filter paper in plates for 3 days at $23^{\circ} \mathrm{C}$. Germinated seed $(n=100)$ were transferred to a strainer ( 28 by 23 by $9 \mathrm{~cm}, \mathrm{~W} \times \mathrm{L} \times \mathrm{H}$ ) lined with a sheet of gauze, and moistened by placing the strainer in a slightly larger container $(28.5$ by 23.5 by $11 \mathrm{~cm}$, $\mathrm{W} \times \mathrm{L} \times \mathrm{H}$ ) containing 1 liter of tap water as the culture medium. Plantlets were grown in a chamber with a photoperiod $\left(120 \mu \mathrm{mol}\right.$ photons $\left./ \mathrm{m}^{2} / \mathrm{s}\right)$ of 14 and $10 \mathrm{~h}$ at 23 and $20^{\circ} \mathrm{C}$, respectively. They were watered with tap water for 7 days and then transferred to $0.5 \times$ Tadano and Tanaka medium without phosphate. The culture medium was replaced every 3 days and, after 10 days, root exudates released into culture medium were adsorbed by activated charcoal using circulation pumps (Akiyama et al. 2005; Yoneyama et al. 2008).

\section{Extraction of germination stimulants from roots and root exudates.}

Extraction of germination stimulants from B. napus roots was adapted from Yoneyama and associates (2008). Roots were ground in liquid nitrogen and the ground material was recovered in $125 \mathrm{ml}$ of ethyl acetate in a glass bottle protected from light, and sonicated for $20 \mathrm{~min}$. After $0.2-\mu \mathrm{m}$ filtration through PTFE (polytetrafluoroethylene) filters (diameter 47 $\mathrm{mm}, 0.2-\mu \mathrm{m}$ pore size) (Whatman $\mathrm{GmbH}$, Dassel, Germany), pellets were extracted further with $125 \mathrm{ml}$ of ethyl acetate overnight at $4{ }^{\circ} \mathrm{C}$. Filtered root extracts were pooled and washed with 1 volume of $0.2 \mathrm{M} \mathrm{K}_{2} \mathrm{HPO}_{4}, \mathrm{pH} 8.3$, with 5 min of shaking. The aqueous phase $(2 \mathrm{ml})$ was sampled for the germination assay on $P$. ramosa seed. The organic phase was dried for $10 \mathrm{~min}$ over anhydrous $\mathrm{MgSO}_{4}, 0.2-\mu \mathrm{m}$-filtered through PTFE, concentrated under reduced pressure (Rotavapor RII; Büchi Labortechnik AG, Flawil, Switzerland), and dissolved in $10 \mathrm{ml}$ of anhydrous hexane. In all, $5 \mathrm{ml}$ was collected in amber glass vials for GC-MS analysis of the extract. The remaining $5 \mathrm{ml}$ was evaporated and extracts were dissolved in $5 \mathrm{ml}$ of acetonitrile and $0.1 \%$ acetic acid (vol/vol) for germination assays and UPLC-MS/MS analysis. Extracts were stored at $-20^{\circ} \mathrm{C}$ before analysis. Concomitantly, root pellets were heated at $60^{\circ} \mathrm{C}$ for $48 \mathrm{~h}$ to determine their dry weight.

To extract germination stimulants from root exudates of hydroponically grown $B$. napus plantlets, activated charcoal was replaced every 3 days and root exudates were collected over 14 days. Root exudates adsorbed on the charcoal were eluted with acetone. After the acetone was evaporated under vacuum, the residue was dissolved in $50 \mathrm{ml}$ of water and extracted three times with $50 \mathrm{ml}$ of ethyl acetate. The ethyl acetate extracts were

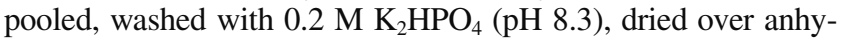
drous $\mathrm{MgSO}_{4}$, and concentrated under vacuum. These crude extracts were stored in sealed glass vials at $4^{\circ} \mathrm{C}$ until use. 
Bioassay-guided RP-HPLC purification of crude extracts.

To identify active compounds, $90 \mu \mathrm{l}$ of crude root or root exudates extracts were fractionated by HPLC performed with a water/acetonitrile gradient applied through a Kromasil 100 C18 $(5 \mu \mathrm{m}, 250$ by $4.6 \mathrm{~mm})$ equipped with a Kromasil C18 guard column ( $5 \mu \mathrm{m}, 10$ by $4.6 \mathrm{~mm}$ ) (Sigma-Aldrich), using a LaChrom Elite HPLC System (Hitachi, Pleasanton, CA, U.S.A.) equipped with an L-2100 pump, L-2200 Autosampler, L-2480 Fluorescence Detector, L-2400 UV Detector, and column thermostat Jetstream 2 Plus. A binary solvent system comprising $\mathrm{A}$, water- $0.1 \%$ acetic acid ( $\mathrm{vol} / \mathrm{vol})$ and $\mathrm{B}$, acetonitrile- $0.1 \%$ acetic acid ( $\mathrm{vol} / \mathrm{vol})$ was used. Separation was performed using a gradient of increasing acetonitrile content with a flow rate of $1 \mathrm{ml} / \mathrm{min}$ and a column temperature of $30^{\circ} \mathrm{C}$. The initial conditions were $40 \% \mathrm{~A}, 60 \% \mathrm{~B}$ and maintained over $5 \mathrm{~min}$. Then the gradient was increased linearly from initial conditions to $0 \% \mathrm{~A}, 100 \% \mathrm{~B}$ over $10 \mathrm{~min}$ and maintained for $5 \mathrm{~min}$. The initial conditions were restored in $2 \mathrm{~min}$ and allowed to equilibrate for $7 \mathrm{~min}$. Then, 1.0 - to $1.5-\mathrm{ml}$ fractions were collected over the separation from 0 to $20 \mathrm{~min}$ and stored at $4{ }^{\circ} \mathrm{C}$ until the germination assay. Absorbance was monitored at $254 \mathrm{~nm}$ and fluorescence at 254- and 350-nm excitation and emission wavelengths, respectively. Chromatographic profiles were plotted using the EZChrom Elite software V.3.1.7 (Scientific Software Inc., San Ramon, CA, U.S.A.).

\section{Extraction of glucosinolate-breakdown products from the $B$. napus rhizosphere.}

Field soil samples (300 to $400 \mathrm{~g} \mathrm{DW}$ ) were harvested during the 2010-11 growing season in the rhizosphere of ES Aliénor and Shakira in an experimental plot located at Saint-Martinde-Fraigneau (France), at the attached buds stage (stages 51 to 53 of the international $\mathrm{BBCH}$ scale depicting B. napus developmental stages) (Lancashire et al. 1991) and during pod maturation (BBCH-71 stage, corresponding to $10 \%$ pods displaying their final size, and $\mathrm{BBCH}-79$ stage, where all the pods reached their final size). Samples were also taken in a neighboring wheat plot as a negative control. Field soil samples were stored at $-20^{\circ} \mathrm{C}$ before extraction. Samples were 1$\mathrm{mm}$ sieved to remove remaining root fragments and were extracted using $500 \mathrm{ml}$ of dichloromethane for $20 \mathrm{~min}$ under shaking. Dichloromethane extracts were filtered through glass fiber filters (diameter $47 \mathrm{~mm}$; Millipore), dried over anhydrous $\mathrm{MgSO}_{4}$ for $20 \mathrm{~min}$ with constant shaking, 0.2- $\mu \mathrm{m}$ filtered through PTFE filters, evaporated under reduced pressure, and dissolved in $5 \mathrm{ml}$ of anhydrous hexane. After collection of 2.5 $\mathrm{ml}$ for GC-MS analysis, hexane was evaporated. Extracts were dissolved in $2.5 \mathrm{ml}$ of acetonitrile- $0.1 \%$ acetic acid ( $\mathrm{vol} / \mathrm{vol})$ for the germination assay.

\section{Germination assay.}

Orobanchaceae seed were surface sterilized for $5 \mathrm{~min}$ in sodium hypochlorite $(12 \%)$, rinsed five times with sterile distilled water and once with $2.5 \mathrm{mM}$ HEPES buffer $(\mathrm{pH} 7.55)$ (adjusted with $\mathrm{KOH}$ ), and suspended in HEPES to a final concentration of seed of $10 \mathrm{~g} /$ liter. In all, $50 \mu \mathrm{l}$ (approximately 100 seeds) were distributed in 96-well plates (Cell Culture Multiwell Plate Cellstar; Greiner Bio-One, Frickenhausen, Germany) and seed were stored 7 days at $21^{\circ} \mathrm{C}$ in the dark as a conditioning period, during which the seed become responsive to germination stimulants. Test solutions for the germination assay were commercial standards, organic or aqueous extracts from roots, root exudates, or field soil samples. Solutions in acetone or acetonitrile were 1:100-diluted in water and aqueous extracts were $0.22-\mu \mathrm{m}$ filtered (PES). Subsequently, $10 \mu \mathrm{l}$ of samples were added to plate wells and volumes were adjusted to $100 \mu \mathrm{l}$ with sterile distilled water, to ensure that each well did not contain more than 1:1000 solvent, unless otherwise mentioned. Seed treated with the synthetic strigolactone GR24 or 1:1000diluted solvents were included as positive and negative controls, respectively. Plates were incubated for germination $\left(21^{\circ} \mathrm{C}\right.$ in the dark). After 4 days, the germinated seed were counted under a stereomicroscope (Olympus SZX10; Olympus Europa GmbH, Hamburg, Germany). Seed were considered as germinated when the radicle protruded out of the seed coat. Each germination assay was repeated at least three times.

For commercial standards, $\mathrm{EC}_{50} \pm$ standard errors were determined from the dose-response curves $(g=f[c]$, where $g=$ germination percentage and $c=$ concentration of the tested compound) and were modeled with a four-parameter logistic curve on at least three independent dilution ranges. Data were computed with SigmaPlot V.10.0 (Systat Software Inc., San Jose, CA, U.S.A.).

\section{Detection and quantification of glucosinolate-breakdown products by GC-MS.}

The analysis of glucosinolate-breakdown products was adapted from Gardiner and associates (1999). Samples $(2 \mu \mathrm{l}$ each) were analyzed using a 5973 mass-selective detector (Hewlett-Packard, Palo Alto, CA, U.S.A.) interfaced to a helium gas chromatograph equipped with a DB-5ms column (30 m by $250 \mu \mathrm{m}, 0.25 \mu \mathrm{m}$ ) (Agilent Technologies, Palo Alto, CA, U.S.A.). Helium was used as carrier gas at a constant pressure of $8.81 \mathrm{psi}$ (nominal initial flow $=1 \mathrm{ml} / \mathrm{min}$ ). Injections were performed in the pulsed splitless mode. Inlet temperature was set to $250^{\circ} \mathrm{C}$. The oven temperature was initially set to $80^{\circ} \mathrm{C}$ and programmed to reach $300^{\circ} \mathrm{C}$. GC-MS transfer line and source were heated at 305 and $230^{\circ} \mathrm{C}$, respectively. The GC-MS system was controlled by HPchem software G1701BA, version B. Extracts were first analyzed in full scan mode in the $\mathrm{m} / z, 50$ to 650 range to identify glucosinolate-breakdown products. Then, for quantification, acquisitions were performed in the selected ion monitoring mode with three to four ions per identified compound. The HP-Chemstation Data Analysis software G1701BA, version $\mathrm{B}$ was used to visualize chromatograms and associated mass spectra. Extracted ion chromatograms were used to characterize the response area of targeted compounds, external standard (4.8 ng of phenanthrene/injection), and calibration standards (3-phenylpropanenitrile, 4-pentenyl isothiocyanate, 2-PEITC, erucin, berteroin, and goitrin). The ratio between the response area of the most abundant fragment from a given compound and phenanthrene (fragment at $\mathrm{m} / \mathrm{z}$ 178) was used for quantification. Nitrile content was expressed relative to a 3-phenylpropanenitrile calibration curve. Contents of individual isothiocyanates and goitrin were expressed relative to the calibration curves of the corresponding standards. Before analysis of RP-HPLC fractions, glucosinolate-breakdown products were extracted with 1.5 volumes of anhydrous hexane.

\section{Strigolactone detection by UPLC-MS/MS.}

UPLC was performed on an Acquity UPLC system (Waters, Millford, MA, U.S.A.). Before analysis of crude root extracts, water- $0.1 \%$ acetic acid ( $\mathrm{vol} / \mathrm{vol})$ was added to ensure strigolactone ionization. Sample $(5 \mu \mathrm{l})$ was separated on an Acquity UPLC BEH C18 column ( 2.1 by $100 \mathrm{~mm}, 1.7 \mu \mathrm{m}$; Waters). The flow rate was $0.6 \mathrm{ml} / \mathrm{min}$ and oven temperature was $40^{\circ} \mathrm{C}$. Eluent A was $0.1 \%$ formic acid in water and eluent B was $0.1 \%$ formic acid in acetonitrile. The following gradient was applied: initial $=5 \% \mathrm{~B}, 0$ to $4 \mathrm{~min}=100 \% \mathrm{~B}$ linear, and 4 to 5 $\min =100 \% \mathrm{~B}$, followed by column wash and reequilibration.

Mass analyses were carried out with a Xevo tandem quadrupole mass spectrometer (Xevo TQ-S; Waters), equipped with an electrospray ionization source used in the positive ion mode. Source parameters were as follows: capillary voltage, 
$2.99 \mathrm{kV}$; cone voltage, $28 \mathrm{~V}$; source offset, $40 \mathrm{~V}$; source temperature, $150^{\circ} \mathrm{C}$; desolvation temperature, $350^{\circ} \mathrm{C}$; desolvation gas flow, 700 liters $/ \mathrm{h}$; collision gas flow, $0.15 \mathrm{ml} / \mathrm{min}$; and nebulizer gas pressure, 7 bars. Nitrogen was used as nebulizer gas and argon was used for fragmentation by collision-induced dissociation. Full-scan spectra were acquired from $\mathrm{m} / \mathrm{z}, 50$ to 500. SRM and Rt were used for strigolactone identification. SRM transitions were optimized in the infusion mode for each strigolactone. Data collection and processing were done with MassLynx software V 4.1. GR24 (50 pg/injection) was used as an external standard.

\section{Co-cultivation experiments.}

Surface-sterilized A. thaliana seed were transferred to 9.4 $\mathrm{cm}$ diameter plates containing MS medium supplemented with $0.6 \%$ (wt/vol) agar. Following stratification for 3 days at $4{ }^{\circ} \mathrm{C}$, seed were incubated at $21^{\circ} \mathrm{C}$ in a growth chamber $(16 \mathrm{~h}$ of light, $8 \mathrm{~h}$ of darkness, and $70 \%$ humidity) for 15 days. Seedlings were then transferred to $15 \mathrm{~cm}$ diameter plates containing MS medium supplemented with $0.6 \%$ (wt/vol) agar. Plates were incubated vertically in the growth chamber for 7 days. Plantlets were then transferred to filter papers, each covered with $20 \mathrm{mg}$ of preconditioned P. ramosa seed placed in cut 15 $\mathrm{cm}$ diameter plates containing a uniform layer of perlite, moistened with $50 \mathrm{ml}$ of $0.5 \times$ Tadano and Tanaka medium. Five plantlets per plate and 10 plates per genotype (Col-0, max3-9, and $\max 4-1$ ) were used.

To study the interaction between $B$. napus and various Orobanchaceae spp., surface-sterilized seed of the highly susceptible cultivar ES Aliénor were placed in a plate between two moistened filter papers and incubated for 7 days in the growth chamber, under the same conditions as A. thaliana. Plantlets were then transferred to filter paper covered with $20 \mathrm{mg}$ of preconditioned Orobanchaceae seed and placed in cut plates containing a uniform layer of rock wool moistened with $50 \mathrm{ml}$ of $0.5 \times$ Tadano and Tanaka medium. Five plantlets per plate and five plates per species (P. ramosa, P. aegyptiaca, O. cumana, $O$. crenata, and $O$. minor) were used.

For co-cultivation, plates were covered with aluminum foil and incubated vertically in the growth chamber for 15 to 21 days. They were watered every 2 days with $10 \mathrm{ml}$ of $0.5 \times$ Tadano and Tanaka medium. At the end of the co-cultivation period, the number of germinated Orobanchaceae seed was evaluated by counting seed from four 2-by-2-cm squares per plate.

\section{Statistical analysis.}

Statistical analyses were performed using SigmaPlot V.10.0. An analysis of variance was performed on the $\mathrm{EC}_{50}$ values with the concentration as the tested factor. Means of at least three independent dilution ranges analyzed by three independent germination assays were tested at $P<0.05$ (Student-NewmanKeuls test). To determine if there were differences between water controls and treatments for the germination assays and for the co-cultivation of Orobanchaceae seed in the rhizosphere of B. napus, a Holm-Sidak multiple comparison test was used, with the treatment condition as the tested factor. Means of at least three independent germination assays or five plates were tested at $P<0.05$. Mean percentages of $P$. ramosa seed germination in the rhizosphere of $A$. thaliana were compared using a $t$ test $(P<$ $0.05)$. All germination percentages were arcsine (square $\operatorname{root}[x]$ ) transformed to normalize the percentages.

\section{ACKNOWLEDGMENTS}

We thank R. Ali (Newe Yaar Research Center, Israel) and Y. Mabrouk (Centre National des Sciences et Technologies Nucléaires [CNSTN], Tuni- sia) for providing $P$. aegyptiaca and $O$. crenata seed populations, respectively; INRA Le Rheu, EURALIS Semences, and SARL Adrien Momont \& Fils for providing B. napus seed; Maïsadour Semences for access to their experimental plot; F.-D. Boyer (CNRS-INRA Gif-sur-Yvette, France) for providing purified strigolactones; D. Bozec (Laboratoire de Biologie et Pathologie Végétales [LBPV]) for help with plant cultivation; Z. Gaudin (LPBV) for advice on co-cultivation and sampling of field soil; and H. Le Gall (LPBV) for technical help. This work was funded through a Fonds de Soutien à la Recherche Semencière Oléagineuse collaborative project. The work from the K. Yoneyama group was supported by a Grant-in-Aid for Scientific Research from Japan Ministry of Education, Science and Culture (KAKENHI) (22-9996) and Program for Promotion of Basic and Applied Researches for Innovations in Bio-oriented Industry. K. Yoneyama was supported by a Japan Society for the Promotion of Science (JSPS) Research Fellowship for Young Scientists.

\section{LITERATURE CITED}

Agerbirk, N., De Vos, M., Kim J. H., and Jander G. 2009. Indole glucosinolate breakdown and its biological effects. Phytochem. Rev. 8:101-120.

Akiyama, K., Matsuzaki, K., and Hayashi, H. 2005. Plant sesquiterpenes induce hyphal branching in arbuscular mycorrhizal fungi. Nature 435:824-827.

Al-Turki, A. I., and Dick, W. A. 2003. Myrosinase activity in the soil. Soil Sci. Soc. Am. J. 67:139-145.

Benharrat, H., Boulet, C., Theodet, C., and Thalouarn, P. 2005. Virulence diversity among branched broomrape $(O$. ramosa L.) populations in France. Agron. Sustain. Dev. 25:123-128.

Bones, A. M., and Rossiter, J. T. 1996. The myrosinase-glucosinolate system, its organization and biochemistry. Physiol. Plant. 97:194-208.

Bones, A. M., and Rossiter, J. T. 2006. The enzymic and chemically induced decomposition of glucosinolates. Phytochemistry 67:1053-1067.

Booker, J., Auldridge, M., Wills, S., McCarty, D., Klee, H., and Leyser, O. 2004. MAX3/CCD7 is a carotenoid cleavage dioxygenase required for the synthesis of a novel plant signaling molecule. Curr. Biol. 14:12321238.

Borek, V., Morra, M. J., and McCaffery, J. P. 1996. Myrosinase activity in soil extracts. Soil Sci. Soc. Am. J. 60:1792-1797.

Brault, M., Betsou, F., Jeune, B., Tuquet, C., and Sallé, G. 2007. Variability of Orobanche ramosa populations in France as revealed by cross infestations and molecular markers. Environ. Exp. Bot. 61:272-280.

Brown, P. D., Tokuhisa, J. G., Reichelt, M., and Gershenzon, J. 2003. Variation of glucosinolate accumulation among different organs and developmental stages of Arabidopsis thaliana. Phytochemistry 62:471-481.

Burow, M., and Wittstock, U. 2009. Regulation and function of specifier proteins in plants. Phytochem. Rev. 8:87-99.

Cheng, D. L., Hashimoto, K., and Uda, Y. 2004. In vitro digestion of sinigrin and glucotropaeolin by single strains of Bifidobacterium and identification of the digestive products. Food Chem. Toxicol. 42:351-357.

de Vega, C., Berjano, R., Arista, M., Ortiz, P. L., Talavera, S., and Stuessy, T. F. 2008. Genetic races associated with the genera and sections of host species in the holoparasitic plant Cytinus (Cytinaceae) in the Western Mediterranean basin. New Phytol. 178:875-887.

Evidente, A., Fernandez-Aparicio, M., Cimmino, A., Rubiales, D., Andolfi, A., and Motta, A. 2009. Peagol and peagoldione, two new strigolactone-like metabolites isolated from pea root exudates. Tetrahedron Lett. 50:6955-6958

Evidente, A., Cimmino, A., Fernandez-Aparicio, M., Andolfi, A., Rubiales, D., and Motta, A. 2010. Polyphenols, including the new peapolyphenols A-C, from pea root exudates stimulate Orobanche foetida seed germination. J. Agric. Food Chem. 58:2902-2907.

Evidente, A., Cimmino, A., Fernandez-Aparicio, M., Rubiales, D., Andolfi, A., and Melck, D. 2011. Soyasapogenol B and trans-22-dehydrocampesterol from common vetch (Vicia sativa L.) root exudates stimulate broomrape seed germination. Pest Manage. Sci. 67:1015-1022.

Fernández-Aparicio, M., Flores F., and Rubiales D. 2009. Recognition of root exudates by seeds of broomrape (Orobanche and Phelipanche) species. Ann. Bot. (London) 103:423-431.

Gardiner, J. B., Morra, M. J., Eberlein, C. V., Brown, P. D., and Borek, V. 1999. Allelochemicals released in soil following incorporation of rapeseed (Brassica napus) green manures. J. Agric. Food Chem. 47:38373842 .

Georgieva, E. A., and Dimitrova-Kostova, Z. S. 1984. Determination of substances with isothiocyanic structure in the meals from the seeds of some plant species provoking the germination of the seeds of the broom rape (Orobanche ramosa L., Orobanche mutelii Schulz.). C. R. Acad. Bulg. Sci. 37:241-244.

Georgieva, E. A., and Dimitrova-Kostova, Z. S. 1986. Determination of substances with isothiocyanic structure in water isolates from of some 
plant species, provoking the germination of the seeds of the broom rape (Orobanche ramosa L., Orobanche mutelii Schulz.). C. R. Acad. Bulg. Sci. 39:127-129.

Gibot-Leclerc, S., Brault, M., Pinochet, X., and Sallé, G. 2003. Rôle potentiel des plantes adventices du colza d'hiver dans l'extension de l'orobanche rameuse en Poitou-Charentes. C. R. Biol. 326:645-658.

Gimsing, A. L., Sørensen, J. C., Tovgaard, L., Jørgensen, A. M., and Hansen, H. C. 2006. Degradation kinetics of glucosinolates in soil. Environ. Toxicol. Chem. 25:2038-2044.

Goldwasser, Y., and Yoder, J. I. 2001. Differential induction of Orobanche seed germination by Arabidopsis thaliana. Plant Sci. 160:951-959.

Goldwasser, Y., Yoneyama, K., Xie, X., and Yoneyama, K. 2008. Production of strigolactones by Arabidopsis thaliana responsible for Orobanche aegyptiaca seed germination. Plant Growth Regul. 55:21-28.

Gomez-Roldan, V., Fermas, S., Brewer, P. B., Puech-Pagès, V., Dun, E. A., Pillot, J. P., Letisse, F., Matusova, R., Danoun, S., Portais, J. C., Bouwmeester, H., Bécard, G., Beveridge, C. A., Rameau, C., and Rochange, S. F. 2008. Strigolactone inhibition of shoot branching. Nature 455:189-185.

Joel, D. M., Chaudhuri, S. K., Plakhine, D., Ziadna, H., and Steffens, J. C. 2011. Dehydrocostus lactone is exuded from sunflower roots and stimulates germination of the root parasite Orobanche cumana. Phytochemistry 72:624-634.

Kim, H. I., Xie, X. N., Kim, H. S., Chun, J. C., Yoneyama, K., Nomura, T., Takeuchi, Y., and Yoneyama, K. 2010. Structure-activity relationship of naturally occurring strigolactones in Orobanche minor seed germination stimulation. J. Pestic. Sci. 35:344-347.

Kirkegaard, J. A., and Sarwar, M. 1998. Biofumigation potential of brassicas. I. Variation in glucosinolate profiles of diverse field-grown brassicas. Plant Soil 201:71-89.

Kohlen, W., Charnikhova, T., Liu, Q., Bours, R., Domagalska, M. A., Beguerie, S., Verstappen, F., Leyser, O., Bouwmeester, H., and RuyterSpira, C. 2011. Strigolactones are transported through the xylem and play a key role in shoot architectural response to phosphate deficiency in nonarbuscular mycorrhizal host Arabidopsis. Plant Physiol. 155:974987.

Lancashire, P. D., Bleiholder, H., Langelüddecke, P., Stauss, R., van den Boom, T., Weber, E., and Witzen-Berger, A. 1991. An uniformdecimal code for growth stages of crops and weeds. Ann. Appl. Biol. 119:561-601.

Lopez-Raez, J. A., Charnikhova, T., Gomez-Roldan, V., Matusova, R. Kohlen, W., De Vos, R., Verstappen, F., Puech-Pages, V., Becard, G., Mulder, P., and Bouwmeester, H. 2008. Tomato strigolactones are derived from carotenoids and their biosynthesis is promoted by phosphate starvation. New Phytol. 178:863-874.

Lopez-Raez, J. A., Matusova, R., Cardoso, C., Jamil, M., Charnikhova, T., Kohlen, W., Ruyter-Spira, C., Verstappen, F., and Bouwmeester, H. 2009. Strigolactones: Ecological significance and use as a target for parasitic plant control. Pest Manage. Sci. 64:471-477.

Mashiguchi, K., Sasaki, E., Shimada, Y., Nagae, M., Ueno, K., Nakano, T., Yoneyama, K., Suzuki, Y., and Asami, T. 2009. Feedback-regulation of strigolactone biosynthetic genes and strigolactone-regulated genes in Arabidopsis. Biosci. Biotechnol. Biochem. 73:2460-2465.

McCully, M. E., Miller, C., Sprague, S. J., Huang, C. X., and Kirkegaard, J. A. 2008. Distribution of glucosinolates and sulphur-rich cells in roots of field-grown canola (Brassica napus). New Phytol. 180:193-205.

Mullin, C. A., Alfatafta, A. A., Harman, J. L., Everett, S. L., and Serino, A. A. 1991. Feeding and toxic effects of floral sesquiterpene lactones, diterpenes and phenolics from sunflower (Helianthus annuus L.) on Western corn rootworm. J. Agric. Food Chem. 39:2293-2299.

Musselman, L. J. 1980. The biology of Striga, Orobanche, and other rootparasitic weeds. Annu. Rev. Phytopathol. 18:463-489.

Parker, A. 2009. Observations on the current status of Orobanche and Striga problems worldwide. Pest Manage. Sci. 65:453-459.

Parker, A., and Riches, C. R. 1993. Parasitic Weeds of the World. Biology and Control. CAB International, Wallingford, U.K.

Pérez-de-Luque, A., Fondevilla, S., Pérez-Vich, B., Aly, R., Thoiron, S., Simier, P., Castillejo, M. A., Fernandez-Martinez, J. M., Jorrin, J., Rubiales, D., and Delavault, P. 2009. Understanding Orobanche and Phelipanche-host plant interactions and developing resistance. Weed Res. 49 (Suppl. 1):8-22.

Pujadas-Salva, A. J. 1999. Species of the family Orobanchaceae parasitic of cultivated plants and its relatives growing on wild plants, in the south of the Iberian Peninsula. Pages 187-193 in: Resistance to Broomrape, the State of the Art. J. I. Cubero, M. T. Moreno, D. Rubiales, and J. C. Sillero, eds. Junta de Andalucia, Andalucia, Spain

Rask, L., Andréasson, E., Ekbom, B., Eriksson, S., Pontoppidan, B., and
Meijer, J. 2000. Myrosinase: Gene family evolution and herbivore defense in Brassicaceae. Plant Mol. Biol. 42:93-113.

Rispail, N., Dita, M. A., González-Verdejo, C., Pérez-de-Luque, A., Castillejo, M. A., Prats, E., Román, B., Jorrín, J., and Rubiales, D. 2007. Plant resistance to parasitic plants: Molecular approaches to an old foe. New Phytol. 173:703-712.

Rubiales, D., and Fernández-Aparicio, M. 2011. Innovations in parasitic weeds management in legume crops. a review. Agron. Sustain. Dev. 2:433-449.

Rumberger, A., and Marschner, P. 2003. 2-Phenylethylisothiocyanate concentration and microbial community composition in the rhizosphere of canola. Soil Biol. Biochem. 35:445-452.

Sakorn, P., Rakariyatham, N., Niamsup, H., and Nongkunsarn, P. 2002. Rapid detection of myrosinase-producing fungi: A plate method based on opaque barium sulfate formation. World J. Microbiol. Biotechnol. 18:73-74

Schwartz, S. H., Qin, X., and Loewen, M. C. 2004. The biochemical characterization of two carotenoid cleavage enzymes from Arabidopsis indicates that a carotenoid-derived compound inhibits lateral branching. J. Biol. Chem. 279:46940-46945.

Seaman, F. C. 1982. Sesquiterpene lactones as taxonomic characters in the Asteraceae. Bot. Rev. 48:121-595.

Sorefan, K., Booker, J., Haurogne, K., Goussot, M., Bainbridge, K., Foo, E., Chatfield, S., Ward, S., Beveridge, C., Rameau, C., and Leyser, O. 2003. MAX4 and RMS1 are orthologous dioxygenase-like genes that regulate shoot branching in Arabidopsis and pea. Gene. Dev. 17:14691474

Spring, O., Kupka, J., Maier, B., and Hager, A. 1982. Biological activities of sesquiterpene lactones from Helianthus annuиs; Antimicrobial and cytotoxic properties; influence on DNA, RNA and protein synthesis. Z. Naturforsch. 37:1087-1091.

Tadano, T., and Tanaka, A. 1980. The effect of low phosphate concentrations in culture medium on early growth of several crop plants. Jpn. J. Soil Sci. Plant Nutr. 51:399-404. (In Japanese).

Thorogood, C. J., Rumsey, F. J., Harris, S. A., and Hiscock S. J. 2008. Host-driven divergence in the parasitic plant Orobanche minor Sm. (Orobanchaceae). Mol. Ecol. 17:4289-4303.

Thorogood, C. J., Rumsey, F. J., and Hiscock S. J. 2009. Host-specific races in the holoparasitic angiosperm Orobanche minor: Implication for speciation in parasitic plants. Ann. Bot. (London) 103:1005-1014.

Umehara, M., Hanada, A., Yoshida, S., Akiyama, K., Arite, T., TakedaKamiya, N., Magome, H., Kamiya, Y., Shirasu, K., Yoneyama, K., Kyozuka, J., and Yamaguchi, S. 2008. Inhibition of shoot branching by new terpenoid plant hormones. Nature 455:185-201.

Virtue, J. G., DeDear, C., Potter, M. J., and Rieger, M. 2006. Potential use of isothiocyanates in branched broomrape eradication. In: Proc. 15th Aust. Weeds Conf. Adelaide, Australia.

Westwood, H. 2000. Characterization of the Orobanche-Arabidopsis system for studying parasite-host interactions. Weed Sci. 48:742-748.

Xie, X., Kusumoto, D., Takeuchi, Y., Yoneyama, K., Yamada, Y., and Yoneyama, K. 2007. 2'-Epi-orobanchol and solanacol, two unique strigolactones, germination stimulants for root parasitic weeds, produced by tobacco. J. Agric. Food Chem. 55:8067-8072.

Xie, X., Yoneyama, K., Kusumoto, D., Yamada, Y., Yokota, T., Takeuchi, Y., and Yoneyama, K. 2008. Isolation and identification of alectrol as (+)-orobanchyl acetate, a novel germination stimulant for root parasitic plants. Phytochemistry 69:427-431.

Xie, X., Yoneyama, K., Kurita, J., Harada, Y., Yamada, Y., Takeuchi, Y., and Yoneyama, K. 2009. 7-oxoorobanchyl acetate and 7-oxoorobanchol as germination stimulants for root parasitic plants from flax (Linum usitatissimum). Biosci. Biotechnol. Biochem. 73:1367-1370.

Xie, X., Yoneyama, K., and Yoneyama, K. 2010. The strigolactone story. Annu. Rev. Phytopathol. 48:93-117.

Yoder, J. I., and Scholes, J. D. 2010. Host plant resistance to parasitic weeds; Recent progress and bottlenecks. Curr. Opin. Plant Biol. 13:478-484.

Yoneyama, K., Yoneyama, K., Takeuchi, Y., and Sekimoto, H. 2007. Phosphorus deficiency in red clover promotes exudation of orobanchol, the signal for mycorrhizal symbionts and germination stimulant for root parasites. Planta 225:1031-1038.

Yoneyama, K., Xie, X., Sekimoto, H., Takeuchi, Y., Ogasawara, S. Akiyama, K., Hayashi, H., and Yoneyama, K. 2008. Strigolactones, host recognition signals for root parasitic plants and arbuscular mycorrhizal fungi, from Fabaceae plants. New Phytol. 179:484-494.

Yoneyama, K., Awad, A. A., Xie, X. N., Yoneyama, K., and Takeuchi, Y. 2010. Strigolactones as germination stimulants for root parasitic plants. Plant Cell Physiol. 51:1095-1103. 